\title{
Über Drusen im Sehnervenkopf.
}

\author{
Von \\ Niels Höeg, \\ Assistent an Prof. Bjerrums Augenklinik (der Universitäts-Augenklinik) \\ in Kopenhagen.
}

Taf. XXXII u. XXXIII, Fig. 1-18.

Die Drusen im Sehnervenkopf bilden ein charakteristisches, aber selten vorkommendes Krankheitsbild, so selten, dass selbst an den grössten Kliniken nur vereinzelte wohlentwickelte Fälle zur Beobachtung kommen, und es ist daher unmöglich, auf Grund der Erfahrungen des einzelnen Beobachters das Krankheitsbild zu beschreiben und dessen klinische Bedeutung zu beurteilen. Indessen finden sich doch in der Literatur eine genügend grosse Anzahl genau untersuchter und beschriebener Fälle, um den Versuch zu gestatten, durch eine Zusammenstellung derselben mit eigenen Beobachtungen eine Darstellung des Krankheitsbildes zu geben und die Bedeutung; die man ihm für die klinische Pathologie des Auges beilegen kann, klar zu machen.

Ich gebe zunächst die Beschreibung der Fälle, die ich selbst zu untersuchen und abzubilden Gelegenheit gehabt habe. Fall II ist an Prof. Edm. Jensens Augenklinik beobachtet, die übrigen Fälle entstammen der Augenklinik des Prof. Bjerrum; für die Überlassung der Fälle spreche ich den beiden Direktoren dieser Kliniken meinen besten Dank aus.

\section{Fall I (Fig. 1 auf Taf. XXXII).}

Marie L., 23jährige Dienstmagd, kam zur Beobachtung am 26.X. 06.

O. s. M. $0,50 s<5 / 9$.

O. d. $\operatorname{Em} . S<5$.

Das Gesichtsfeld des linken Auges ist temporal bis zum blinden Fleeke, nasal bis zu $30^{\circ}$ eingeengt, während es oben und unten seine normale Ausdehnung hat. Das Gesichtsfeld des reehten Auges ist normal. Die Untersuehung wurde nur mit grossem ${ }^{1}$ ) weissem Objekt (Gesichtswinkel 9/:00) vorgenommen.

1) Überall, wo in meinen Krankengeschichten von grossen und kleinen 
Beide Augen sind äusserlich normal, brechende Medien vollständig klar.

Ophthalmoskopie: Oc. sin. (Fig. 1, aufrechtes Bild): Die Papille ist etwas vergrössert und geschwollen, ihre Grenzen verwischt. Der nächst umgebende Teil des Augengrundes ist leicht depigmentiert. In der Substanz der Papille finden sich zahlreiche, weisslich glänzende, teils konglomerierte, teils isolierte Gebilde von verschiedener Grösse, die grössten die Breite der Hauptvenen im Durchmesser etwas übertreffend. Sie finden sich sowohl am Rande der Papille als innerhalb dieser und scheinen teils oberflächlich, teils tiefer im Gewebe gelagert zu sein. Ihre Farbe ist weisslieh, leicht bläulich, von einem halbdurchseheinenden, porzellan- oder milehglasähnlichen Charakter; sie reflektieren das Lieht gleichartig von ihrer ganzen Oberfläche, und gewinnen dadurch ein flaches Aussehen wie Schuppen, ein Aussehen, das sich doch bei Untersuchung ihres parallaktischen Verhaltens ändert, indem die grössten sich dadurch als deutlich prominierende Knollen und Knötchen zeigen; namentlich prominiert eine Gruppe von drei Kügelchen, die im temporal-unteren Quadrant der Papille liegen.

Die Gefässe sind von natürlicher Farbe, normalem Kaliber und Verlauf und sind nirgends von den Gebilden überlagert.

Der übrige Augengrund zeigt keine Abnormitäten.

Oe. dext.: Die Papille ist auch hier leicht geschwollen mit verwischten Grenzen und entfärbt, doch sind diese Symptome im ganzen weniger ausgeprägt als im linken Auge. Es finden sich keine Drusen; nur in der oberen Hälfte der Papille bildet ein kleiner glitzernder Punkt vielleicht eine Andeutung solcher.

Ubriger Augengrund normal.

Anamnestische Data fehlen.

Es scheint mir unzweifelhaft, dass es sich in diesem Falle um doppelseitige abgelaufene Neuritis (oder Stauungspapille) mit sekundärer Drusenbildung handelt. Der Zustand der rechten Papille, in der fast keine Drusen zu beobachten waren, und die peripapilläre Entfärbung des Augengrundes sprechen hierfür.

Die Herabsetzung des Sehvermögens und die Einschränkung des Gesichtsfeldes lassen sich wohl zwanglos auf Rechnung des primären Sehnervenleidens schreiben; ob die Drusen hieran mitschuldig sind, ist unmöglich zu entscheiden.

Fall II (Fig. 2 auf Taf. XXXII),

Anna J., 36jährige Frau, stellte sich am 19. XI. 06 in Prof. Edm. Jensens Augenklinil vor.

Bei der ophthalmoskopischen Untersuchung wurde am reehten Ange

weissen Objekten die Rede ist, handelt es sich beim grossen Objekt um gewohnliche Perimeteruntersuchung, beim kleinen Objekt um Bjerrums Gesichtsfelduntersuchung; der Gesichtswinkel des Objekts ist (nach Bjerram) durch seinen numerischen. Wert angegeben, also durch das Verhältnis des Objektdiameters zum Abstand des Objekts vom Ange (beide Grössen in mm ausgedrückt). 
ein höchst auffallender Fund konstatiert (Fig. 2, aufrechtes Bild): Vor dem nasalen Teil der Papille liegt im Glaskörper ein weisses, stark glänzendes und glitzerndes Gebilde, das einen Flächenraum von ungefähr $11 / 2$ Papillengrösse und von umgekehrter Birnform deckt. Sein Rand ist überall ganz scharf, und liegt deutlich über dem Augengrund hervorgehoben, was überall durch parallaktisehe Verschiebung und Refraktionsdifferenz (ungefähr $1 D$ ) leicht zu konstatieren ist; es findet sich also keine sichtbare Verbindung mit der Papille oder mit dem Augengrund. Die Vorderfläche ist noch mehr hervorgewölbt und hebt sich bis zu 4 Dioptrien über den Augengrund.

Das Gebilde ist von einer ausserordentlich grossen Menge kleiner, weisslicher, stark glänzender, halbklarer Kügelchen zusammengesetzt, welche überall von ungefähr gleicher Grösse (Durchmesser ungetähr gleich der Breite einer Hauptvene) und Aussehen sind; sie scheinen von einem ïberall gleichartigen, homogenen, klaren oder halbklaren Stoffe gebildet zu sein. Nirgends sieht man Flecken von mehr opakem, mehr weisslichem oder mehr glänzendem Charakter; es finden sich also keine Zeichen von degenerierten oder verkalkten Partien des Gebildes. Die Form der Kügelchen scheint sehr regelmässig zu sein, was man namentlich an der Kontur des Gebildes wahrnimmt, wo die einzelnen Kügelchen sich als regelmässig cirkulär begrenzte Vorsprünge präsentieren. Der Lichtreflex nimmt den Rand der Kügelchen ein, was man auch am deutlichsten an den Konturen sieht; auf der Vorderfläche bilden die Lichtreflexe hauptsächlich ein mehr oder weniger dentlich ausgeprägtes Muster von leuchtenden polygonalen oder runden Figuren.

Art. nas. sup. ist eine kurze Strecke vom Gebilde emporgehoben und liegt also hier auf der Oberfläche desselben, ganz nahe und parallel mit dessen temporal-oberen Rande. Sie gibt einen dünneren Ast $a b$, der in die Substanz des Gebildes eintaucht, nahe dem nasal-oberen Rande wieder hervorkommt und dann über diesen Rand sich herabkrümmend den Augengrund erreicht, um sich in diesem fortzusetzen. Die übrigen Gefässe haben alle einen ganz natürlichen Verlauf.

Der sichtbare Teil der Papille ist vollständig normal; die Farbe, die Begrenzung, die Gefässe, die kleine physiologische Excavation geben nicht den leichtesten Anhalt für die Annahme irgend einer Anomalie. Ebenso ist auch der ubrige Angengrund ganz normal. Der Glaskörper, speziell der das Gebilde nächst umgebende Teil desselben, ist vollkommen durchsichtig, so dass alle Einzelheiten vollkommen scharf wahrzunehmen sind.

Die ophthalmoskopische Untersuchung des linken Auges zeigt ganz normale Verhältnisse und nicht die leichteste Andeutung einer ähnlichen Anomalie.

Die übrige objektive Untersuchung beider Augen bietet gar keine Anomalien mit Ausnahme einer leichten Conjunctivitis.

Beide Augen sind emmetropisch and haben normale Sehschärfe $(S=6 / 6)$.

Das Gesichtsfeld des rechten Auges weist bei Untersuchung mit grossem und kleinem weissem Objekt (Gesichtswinkel ${ }^{20} / 300$ und $5 / 2000$ ) eine kleine sektorförmige Einengung auf, die genau temporal liegt, und für das grosse Objekt ungefähr $20^{\circ}$, für das kleine ungefähr $10^{\circ}$ betrifft. Der blinde Fleck ist vergrössert, erstreckt sich horizontal von $15^{\circ}$ bis an $27^{\circ}$, von gleicher Grösse bei Untersuchung mit grossem und kleinem Objekt. Das 
Gesichtsfeld des linken Auges ist für grosses und kleines weisses Objekt normal.

Anamnestisch teilt die Patientin folgendes mit: sie stammt aus gesunder Familie; als 6-7jähriges Kind hat sie die Variolae durchgemacht, weiss ja doch nichts näheres über diese Krankheit (sie hat keine Pockennarben); 14 Jahre alt hatte sie Febris typhoidea. Später ist sie immer mit Ausnahme leichter Yerdauungsbeschwerden vollständig gesund gewesen; sie hat eine gesunde Tochter von 10 Jahren; sonst hat sie nie geboren und anch nicht abortiert. Sie hat niemals an starkem oder anhaltendem Kopfweh oder andern Symptomen vom Zentralnervensystem gelitten. Sie gibt an, dass ihre Augen niemals krank und immer gut funktionierend gewesen sind.

Eine wiederholte Untersuchung nach Verlauf eines halben Jahres (am 15. V. 07) zeigt keine Veränderung des Bildes; es hat kein sichtbares Wachstum stattgefunden, und das Gebilde präsentiert sich in jeder Beziehung ganz wie bei der ersten Beobachtung.

Dass es sich hier um einen Fall von gleicher Natur als die bisher beschriebenen Drusen des Sehnervenkopfes handelt, ist nicht zu, bezweifeln; wohl findet sich in der Literatur kein Fall, in welchem - wie hier - die sichtbare Drusenmasse ausschliesslich im Glaskörper liegt, aber die Ähnlichkeit mit einigen der veröffentlichten Fälle, namentlich Niedens, ist doch unverkenmbar. Als Ausgangspunkt des Gebildes muss also die Papille - oder möglicherweise die Nervenfaserschicht der benachbarten Retina - angenommen werden. Dass die Glaslamelle der Chorioidea die Ausgangsstelle sein könnte, ist ganz unannehmbar; die Glaslamelldrusen sind niemals bloss annäherungsweise in dieser Weise konglomeriert beobachtet worden, und anch das Alter der Patientin und die Intalktheit des übrigen Augengrundes sprechen dagegen.

Dass der sichtbare Teil der Papille vollkommen normal aussieht, findet die natürliche Erklärung in der Annahme, dass die Drusenbildung in den oberflächlichsten Schichten der Papille stattgefunden habe, und dass die Drusen sehr frühe, ohne sich in dio Papillensubstanz zu infiltrieren, in den Glaskörper hineingebrochen seien, und hier für ihre Weiterentwicklung einen geringeren Widerstand als im festeren Papillengewebe gefunden haben. Mit der Annahme leichterer Wachtumsbedingungen stimmt auch die Regelmässigkeit und Gleichartigkeit der einzelnen Elemente und der Mangel an Degenerations- oder Verkalkungszeichen.

Die wichtige Frage, ob in diesem Falle eine andere Erkrankung des Sehnerven als Ursache der Drusenbildung vorliege, muss nach aller Wahrscheinlichkeit verneinend beantwortet werden; die objektive Untersuchung gibt ja nicht den geringsten Anhaltspunkt für die An-. 
nahme eines primären Leidens, speziell ist der sichtbare Teil der Papille von vollkommen natürlichem Aussehen; die einzige vorliegende Funktionsanomalie, der kleine Gesichtsfelddefekt, scheint leicht erklärbar als Folge einer mechanischen Läsion der Nervenfasern durch den Stiel der Drusenmasse. Die Vergrösserung des blinden Fleckes ist nur als Schatten der Drusenmasse aufzufassen, wie es auch aus der Gleichheit der Grösse bei Untersuchung mit grossem und kleinem Objekt hervorgeht.

Merkwürdig ist in diesem Falle die Einseitigkeit der Anomalie; sonst pflegt bei starker Drusenentwicklung im einen Auge jedenfalls beginnende Drusenbildung im andern vorzuliegen. Man konnte wohl annehmen, dass die Drusengeschwulst wegen des geringen Wachstumswiderstandes des Glaskörpers sich relativ schnell entwickelt hätte, aber die Beobachtungsdauer von einem halben Jahre, in welchem das Gebilde unverändert blieb, zeigt, dass das Wachstum nun abgeschlossen ist oder jedenfalls sich nur ganz langsam fortsetzt. Bei unserer sehr mangelhaften Kenntnis von der Pathogenese der Drusen ist es eben anzunehmen, dass die unbekannte Ursache der Drusenbildung in diesem Falle einseitig ist.

\section{Fall III (Fig. 3 u. 4 auf Taf. XXXII).}

Bengta C., 34jährige Weberin, stellte sich am 13. VIII. 07 vor.

Beide Augen emmetropisch, Sehschärfe normal. Das Gesichtsfeld ist für grosse weisse Objekte (Gesichtswinkel $20 / 300$ ) beiderseits normal; für kleine weisse Objekte $\left(\left.{ }^{5}\right|_{2000}\right)$ ist es am rechten Auge ebenfalls normal, während es am linken im Vergleich mit dem des rechten im ganzen leicht konzentrisch eingeengt ist und im nasal-unteren Meridian eine kleine weitere Einengung zeigt (ungefähr 100).

Beide Augen sind äusserlich normal, die breehenden Medien klar.

Ophthalmoskopie (0. d. Fig. 3, o. s. Fig. 4, aufrechtes Bild): Die Papille, die an beiden Augen etwa das gleiche Aussehen darbietet, ist mässig geschwollen (bis an 2,0 D Refraktionsunterschied), ihre Grenzen sind etwas verwischt. Die nasale Hälfte der Papille scheint von einer grossen Menge kleinerer und grösserer weisslicher, rundlicher Gebilde ausgefüllt, die ziem. lich dicht beisammen gelagert sind. Ihre Farbe ist glänzend weiss, der Rand ist in der Regel etwas heller als die Mitte. Die grössten haben einen Durchmesser ungefähr gleich dem der grossen Venenäste. Sie sind nicht ganz scharf abgesetzt, scheinen hauptsächlich tiefer im Papillengewebe zu liegen, und nicht über dessen Oberfläche zu prominieren.

Die temporale Papillenhälfte zeigt nur Andeutungen von solchen $\mathrm{Ge}$ bilden; die Farbe der Papille ist hier gleichartig dunkelgraurötlieh, mehr dunkel und minder rötlich als gewöhnlich.

Die Gefässe verhalten sich an den beiden Augen etwas verschieden. Am rechten Auge findet sich eine Vena optico-ciliaris, die am nasal-unteren 
Rande hervorkommend in temporaler Richtung leicht S-fồmig gekrümmt verläuft, um hinter der A. papill. inf. sich mit der Vena temp. inf. zu verbinden; kurz vor dieser Verbindung empfängt sie zwei schmale Äste von unten and von unten-nasal. Die Dicke und die Farbe der V. optico-ciliaris ist ungefähr wie die der V. temp. inf., doch hat sie keinen Reflexstreif. Durch leichten Druck auf den Bulbus entsteht Venenpuls sowohl an der V. optico-ciliaris als an den übrigen Venen. Die oberen Venen sind leicht geschlängelt, doch nicht mehr als es oft unter physiologisehen Verhältnissen wahrgenommen wird.

Am linken Auge sind sümtliche Venen stark geschlängelt und vielleicht leicht erweitert; V. temp. inf. und eine kleine V. macularis bilden am Rande der Papille schleifenartige Schlingen; eine optico-ciliare Vene findet sich hier nicht.

Die Arterien bieten — an beiden Augen - keine Abnormitäten dar.

Anamnese: Stirps sana. Patientin ist immer gesund gewesen, hat niemals Zeichen eines Leidens des Zentralnervensystems dargeboten; sie gibt an, dass sie niemals krankhafte Symptome an den Augen bemerkt hat.

In diesem Falle sehe ich es als wahrscheinlich an, dass die Trübung des Papillengewebes, die Schlängelung der Venen am linken und die Vena optico-ciliaris am rechten Auge als Zeichen eines fruheren Leidens des Nervus opticus anzunehmen sind, das, ohne die Funktion wesentlich zu schädigen, zurückgegangen ist. Sowohl die Schlängelung der Venen als die optico-ciliare Vene lassen sich als Folgen eines Cirkulationshindernisses im Gebiete der V. centr. retinae auffassen, und indem man annimmt, dass das Cirkulationshindernis am rechten Auge durch die Entwicklung der optico-ciliaren Anastomose beseitigt worden ist, versteht man, warum die Venen hier nicht geschlängelt sind. Möglicherweise verdankt dieses Auge der Entstehung der optico-ciliaren Vene seine völlig intakte Funktion.

Man könnte vielleicht auch meinen, dass die Drusen das primäre Leiden wären, und dass sie durch Druck auf die Zentralvene das Cirkulationshindernis verursacht hätten. Es findet sich aber kein einziges früheres Beispiel von einer durch die Drusen entstandenen selbst leichten Cirkulationshemmung, auch nicht, wenn die Drusen eine ganz bedentende Entwicklung erreicht haben, und es ist also wohl anzunehmen, dass der Wachstumsdruck der Drusen gar nicht genügt, um eine merkbare Cirkulationshemmung zu verursachen.

Fall IV (Fig. 5 u. 6 auf Taf. XXXII).

Anna J., 53jährige Frau, kam zur Beobachtung am 14. VIII. 07.

Beide Augen leicht hypermetropisch (o. d. H. 1,00, o. s. H. 1,50) mit normaler Sehschärfe. Das Gesichtsfeld, mit grossem (20/300) und kleinem $(5 / 2000)$ weissem Objekt gemessen, weist keine Defekte auf, Die Augen sind äusserlich normal, brechende Vedien klar. 
Ophthalmoskopie (o. d. Fig. 5, o. s. Fig. 6, aufrechtes Bild): An beiden Augen sind die Papillengrenzen leicht verwischt, die Farbe der Papillen ist wie im vorigen Falle etwas dunkler grauartig und minder rötlich als gewöhnlich. In der Papillensubstanz findet sich beiderseits eine Gruppe rundlicher weisslicher Gebilde von verschiedener Grösse; im rechten Auge nehmen sie den oberen-nasalen Quadranten ein, im linken finden sie sich im nasalen Papillenteil. Sie gleichen den Gebilden im vorigen Falle, doch sind sie minder auffallig, nicht so glänzend, von einer mehr mattweissen Farbe. Die Papillen sind nicht gesehwollen, die Gefässe verhalten sich ganz normal.

Der iibrige Augengrund bietet gar keine Anomalien dar.

Anamnese: Patientin hat vorigen Winter an Influenza, Pneumonie und Bronchitis gelitten und wird gegenwärtig wegen eines Herzfehlers behandelt. Sie hat seit ibrer Kindheit fast immer an starken und anhaltenden Kopfsehmerzen gelitten, namentlich in den späteren Jahren. Sie hat einmal geboren (das Kind gesund), leugnet Miss- und Totgeburten. Sie befindet sich jetzt im Klimakterium.

$\mathrm{Ob}$ hier der Folgezustand eines früheren ohne Verletzung der Funktion verlaufenen Optikusleidens vorliegt, oder ob die Drusen sich in einer sonst normalen Papille entwickelt haben, lässt sich wohl nicht mit Sicherheit entscheiden; die Verwischung der Papillengrenzen und die grauliche Farbe des Gewebes waren jedenfalls nicht mehr ausgeprägt, als man unter normalen Verhältnissen sehen kann.

\section{Fall V.}

Christine H., 61 jährige Frau, am 12. IX. 07.

Am linken Auge finden sich am nasalen Papillenrande fünf kleine weissliche Gebilde, teils innerhalb, teils ausserhalb des Randes, das eine den Pigmentsaum verdeckend.

Mit Ausnahme eines kleinen (senilen?) Conus temp. gibt die Untersuchung des Auges sonst keine Anomalien.

Rechtes Ange normal.

Patientin ist immer gesund gewesen.

$\mathrm{Ob}$ die kleinen weissen Plaques in diesem Falle von derselben Natur sind wie in den andern, scheint mir unsicher. Doch stimmt ihr Aussehen gut mit der Beschreibung beginnender Drusenentwicklung in mehreren Publikationen überein. Für die Annahme eines sonstigen Leidens des Sehnerven - vielleicht mit Ausnahme leichter seniler Veränderungen - liegt gar kein Grund vor.

Ich gebe hiernach eine Übersicht über die bisher in der Literatur mitgeteilten Fälle und die Anschauungen, die über ihre Pathogenese und klinische Bedeutung hervorgebracht sind. 
Die Drusen des Sehnervenkopfes gehören zu den Anomalien des Auges, bei denen der anatomische Nachweis der klinischen Erkennung des Krankheitsbildes vorausgegangen ist, da die zwei ersten Mitteilungen dieser Art über mikroskopisch untersuchte Fälle berichten.

H. Müller $(1,1858)$ ist der erste, der einen Fall von der bis dahin unbekannten Krankheit mitteilt:

In beiden Augen eines 75 jährigen fast gänzlich blinden Mannes fand er, ausser den Zeichen von exquisit getigerter Netzhaut, eine beträchtliche Konkretion an der Durehtrittsstelle des Sehnerven; dieselbe bildete eine Zone von 0,4 bis $0,6 \mathrm{~mm}$ Höhe in der Gegend der Lamina cribrosa und bestand aus grösseren und kleineren gelblichen sandähnlichen Körnehen von drusiger Bildung, die in einer geschichteten organisehen Grundlage kohlensauren Kalk enthielten; Jod bewirkte daran bloss eine gelbe Färbung.

Sowohl die Beschreibung der Konkretion als das beigefügte Bild machen es zweifellos, dass es sich um dieselbe Anomalie handelt, die später als Drusen des Sehnervenkopfes beschrieben wird. kretionen:

Erst 10 Jahre später folgte die nächste Mitteilung über ähnliche Kon-

Iw a n off $(2,1868)$ besehrieb sie als Ablagerungen von geschichteten Konkretionen nach innen von der Lamina eribrosa in der Substanz der Papille. Die Konkretionen - auf Längsschnitte zu beiden Seiten der Zentralgefässe gelagert - bestanden aus einer grösseren Anzahl ganz kleiner, konzentrisch geschichteter, Amyloidkörperchen ähnlicher Körner, welche jedoch keine Amyloidreaktion gaben, und sie liessen sich durch die Sehnervensubstanz bis zur Glaslamelle der Chorioidea hin verfolgen. I wan off nimmt daher als wahrseheinlich an, dass es sieh um drusige Ablagernngen der Glaslamelle der Aderhaut handelt, die vom Rande der letzteren aus in den Sehnerven eingedrungen sind.

Er fand diese Veränderung an 6 Augen (von 5 Individuen). In einem Fall war das Sehvermögen während des Lebens völlig intakt, ein anderer betraf einen Geisteskranken mit absoluter Amaurose, in den übrigen waren die Augen wegen Verletzungen oder fremder Körper enucleiert.

Gleichzeitig (in der Diskussion nach Iwan offs Vortrag) gibt Liebreich $(3,1868)$ die erste ophthalmoskopische Beschreibung der Anomalie:

In Fällen, in denen vielleicht gar keine Sehstörung vorhanden ist, sieht man, am besten bei der Untersuchung im aufreehten Bilde, in der Papille, hinter der Nervenfaserstreifung, einzelne das Licht eigentümlich reflektierende Körper, welche bisweilen auch etwas über die Chorioidalgrenze hinaus gehen.

In Graefe-Saemischs Handbuch der gesamten Augenheillkunde sehliessen Wecker $(4,1876)$ und Leber $(5,1877)$ sieh den Anschaumngen I wan offs über die Natur der Konkretionen an, und es war hiernach ganz matürlich, dass die folgenden Autoren, die ophthalmoskopische Beobachtungen dieser Anomalie mitgeteilt haben, ihre Herkunft aus der Glaslamelle der Chorioidea nicht in Zweifel zogen. 
Nieden $(6,1878)$ beschreibt einen Fall von sehr starker Drusenbildung im Umfange des intraokularen Sehnervenendes an beiden Augen eines 14jährigen Mädchens mit exquisiter Retinitis pigmentosa. Die Drusen, die den Rand der Papille einnahmen, bildeten hier wulstartige Excrescenzen von lichtblau-grauer Farbe, die als halbkuglig gestaltete Buckeln, besetzt mit unzähligen kleinen glitzernden und blitzenden, fast Fettröpfehen ähnelnden Knötchen, weit in den Glaskörperraum hineinragten; einzelne Retinalgefässe waren von den Excrescenzen gedeckt, aber nicht komprimiert; der zentrale Teil der Papille war intakt. Das Bild war an beiden Augen etwa dasselbe, im linken Auge etwas stärker entwickelt.

Die Herabsetzung der S. und der übrigen Funktionen der Augen war nur auf Rechnung der Retinitis pigmentosa zu setzen.

[Diesen Fall hat Nieden in seiner weiteren Entwieklung verfolgt und gab später $(21,1889$ und 33,1900$)$ die Beschreibung der mehr vorgesehrittenen Entwicklungsstadien desselben, worauf wir zurückkommen werden.]

Jany $(7,1879)$ beschrieb einen Fall von Drusenbildung an beiden Papillen eines 37 jährigen Mannes, dessen Augen im übrigen keine Anomalien darboten. Die Papillen waren hier in toto vergrössert und stark gesehwollen, ungleichmässig höekrig und bucklig hervorgetrieben und fielen gegen die Nachbarsehaft steil ab; die kuglig-drusigen Vorsprünge, welche namentlich die Randzone einnahmen, gaben einen eigentïmlichen weisslichen, stellenweise perlmutterartig glänzenden Reflex; die Retinalgefässe waren nicht vẹrdeckt, sondern machten beim Übersehreiten der Höcker grosse Bogen.

S. o. d. ${ }^{6} / 6,0$. s. ${ }^{6} / 9$. Patient litt seit länger als einem Jahr an Kopfschmerzen.

Oeller $(8,1879)$ wendet sich auf Grundlage der mikroskopischen Untersuchung eines Auges mit Chorioretinitis pigmentosa und Drusenbildung in der Papille entschieden gegen die bisher unbestrittene Iw an offsehe Auffassung. Das Auge, das in frühester Kindheit der jetzt $37 j a ̈ h r i g e n ~ P a t i e n t i n$ von einem Peitschenhieb getroffen war, wurde wegen wiederholt auftretender Entziindungen und halbseitigem Kopfweh enucleiert. Oeller fand in einer Reihe von Schnitten nirgends einen Zusammenhang zwischen den Drusen und der Glaslamelle und meint mit Sicherheit aussehliessen zu können, dass sie von der Glaslamelle stammen; er hält es dagegen für möglich, dass sie, trotz fehlender Jodreaktion, mit amyloiden Konkretionen verwandt seien, und dass sie sich vielleicht ans Myelin bilden. Er hat diese Konkretionen auch in einem Falle von totaler Netzhautablösung in der bereits bindegewebig degenerierten Faserschicht der abgelösten Retina angetroffen.

Trotz dieser entschiedenen Aussprüche gegen die frühere Auffassung der Sebnervendrusen als von der Glaslamelle entstammender Gebilde, und trotz der geringen Beweiskraft, die in der Iwanoffsehen Mitteilung zu finden war und in der die einzige stütze für diese Auffassung bestand, scheint dieselbe doch auch in den folgenden Jahren alleinherrschend zu sein. Fs folgte nun eine Reihe von klinischen Beobachtungen: 
W. Stood $(9,1883)$ teilte 2 Fälle mit (Fig. 11 a. 12, Taf. XXXIII):

1. Bei einem 31jährigen Manne mit chronischer Myelomeningitis waren die Papillengrenzen beiderseits verschwommen, die Papille wenig geschwollen, blass, mit diskreten eingesprenkelten, fettig glänzenden Körnchen; am äusseren Rande rechts fanden sich einige streifige Exsudate. 8 Jahre später waren die Papillen ungewöhnlich blass, unregelmässig begrenzt, und zeigten glänzende, weisse, stark reflektierende, rundliche Flecken, die am äusseren-unteren Rande schärfer umschrieben waren und dabei aus der Papillensubstanz als runde kleine Höcker hervortraten. Die Gefässe waren nicht alteriert; S. war normal, Gesichtsfeld eingeengt; Lichtsinn herabgesetzt. Diagnose: descendierende Neuritis mit Drusenbildung.

2. Bei einer 17jährigen Patientin zeigte die temporale Papillenhälfte beiderseits an der konvexen Seite in ihrer ganzen Ausdehnung kleine, stark glänzende, weisse, rundliche Hervorragungen, die als kuglige Höckerchen etwas in den Glaskörperraum hineinragten und den Eindruck von aufgelagerten Glasperlen machten; sie waren am rechten Auge weniger zahlreich als am linken. Die Papillen waren übrigens blass mit verwaschenen Grenzen; die Sehschärfe war $\left.{ }^{6}\right|_{18}$ 0. d., $\left.{ }^{6}\right|_{36} \quad$ o. s. (Strabismus converg.), das Gesichtsfeld eingeengt, der Lichtsinn herabgesetzt. Diagnose: Abgelaufene Neuritis optica mit Drusenbildung.

G. Lawson $(10,1883)$ fand in einem Falle von syphilitischer Chorioretinitis im rechten Auge nach oben aussen von der Papille ein grosses weisses, durchscheinendes, aus sehr zahlreichen Körperchen zusammengesetztes, 2,5 $D$ prominierendes Gebilde, und am Rande der Papille eine ähnliche Ablagerung. Im linken Auge fanden sich zwei kleinere ähnliche Gebilde.

Story $(11,1883)$ sah in einem Falle von anormalem Verlauf der Retinalarterien - wahrseheinlich als Folge früherer Obliteration der A. nas. sup. - zwei grauweisse kreisförmige Fleckchen in der temporalen Hälfte der Papille, und er erwähnt einen Fall Bensons, in dem ähnliche Körperchen die Papille verdeckten and auch ausserhalb ihres Randes zu beobachten waren.

H. Schäfer $(12,1884)$ fand im enucleierten Bulbus eines 13jährigen Mädchens, dessen anderes Auge gesund war, ausser den Zeichen von Iridochorioiditis suppurativa, im Sehnervenkopfe, der ein wenig geschwollen und infiltriert war, nahe der Lamina cribrosa ganz vereinzelt eingelagerte, verschieden grosse, rundliche, geschichtete, drusenartige Konkretionen, die von den Tinktionsmitteln nur ganz schwach sich färbten.

Masselon (13, 1884) sah bei einem 57jährigen Manne Gruppen von hyalinen Körperchen an beiden Papillen; die Augenhintergründe waren ìm übrigen gesund und die Sehschärfe normal.

Ausserdem hat er peripapilläre Drusen bei Glaukoma chronicum gesehen und meint, dass sie für die Pathogenese des Glaukoms Bedeutung haben können, indem sie vielleicht die hinteren Filtrationswege des Auges obstruieren können.

Hirschberg $(14,1884)$ hat bei Retinitis pigmentosa die leuchtenden Punkte am Umfange des Sehnerven mehrmals beobachtet, aber nie so hochgradig wie in Niedens Fall. 
Hirschberg und Birnbacher $(15,1885)$ fanden anatomisch traubenartig konglomerierte, glasige, aus kugelförmigen Körperchen zusammengesetzte Massen in der Nähe der Lamina cribrosa in beiden Augen eines 60jährigen, an Apoplexia cerebri gestorbenen Mannes, der an hochgradiger Arteriosklerose und interstitieller Nephritis gelitten hatte. Beide Sehnerven waren der Sitz eines auf die vorderen Absehnitte beschränkten Prozesses chronischer Staunng. Die Konglomerate zeigten nicht Amyloidreaktion, sie färbten sich intensiv mit Eosin und nahmen bei Pikrokarmintinktion eine kräftige rote Färbung an.

R. Ancke $(16,1885)$ veröffentlichte aus Hirschbergs Augenklinik mehrere Fälle von Retinitis pigmentosa mit Drusenbildung am Papillenrande, in allen Fällen doch in geringem Grade.

1.-2. Bei 5 Geschwistern waren drei von Retinitis pigmentosa befallen. Bei 2 dieser (einem 16jährigen und einer 19jährigen) fanden sich zugleich Drusen am Rande und im Parenchym der Papille, beim Bruder doch nur deutlich am linken Auge.

3.-4. Bei einer andern Familie, in der auch von 5 Geschwistern 3 an Retinitis pigmentosa litten, fanden sich gleichfalls bei zweien (einer 18jährigen und einer 16jährigen) Drusen auf oder nahe den Papillen.

Die Drusen werden als kleine glitzernde Pünktehen, hellglänzende Stellen oder runde prominierende, besonders am Randteil deutlich glänzende Figuren beschrieben.

Remak (17, 1885): Bei einem 52 jährigen Patienten, dessen reehtes Auge infolge einer Stichverletzung im 2. Lebensjahre hochgradig phthisisch gesehrumpft and blind war, fand sich an linken Auge - nach einer Kataraktextraktion - ausser atypischer Chorioretinitis pigmentosa folgender ophthalmoskopischer Befund. An der Stelle der Papille erblickt man ein Gebilde, bestehend aus 5-6 rundlichen und länglichen Konglomeraten von intensiv bläulichweisser Farbe, die durch leichte Einschnürungen andentungsweise voneinander getrennt sind. Sie sind in einem Bogen angeordnet, dessen Konkavität gegen die Maenlagegend gerichtet ist; ihre Oberfläche iiberragt das Retinalniveau ziemlich bedeutend; auf ihrer Oberfläche sind einige ganz feine Gefässe wahrzwnehmen, aber von einer gemeinsamen Ursprungsstelle derselben ist nichts zu sehen.

$S=$ Finger auf ungefähr $11_{2} \mathrm{~m}$, Gesichtsfeld hochgradig eingeengt.

Wedl und Boek $(18,1886)$ bilden Schnitte ab von der Papille eines 24 jährigen, an Hydrocephalus chron. gestorbenen Mannes, der an Neuroretinitis mit nachfolgender Atrophie gelitten hatte; es fanden sich grosse verkalkte Massen aus konglomerierten Knötehen zusammengesetzt.

Gessner $(19,1888)$ fand bei einem Manne mit Enophthalmus traumaticus (nach Läsion vor $1^{1} / 2$ Jahren) am änsseren Rande der sonst normalen Papille drei perimutterartig glänzende prominente Gebilde. $S=1 / 8$.

W. Rath $(20,1888)$ fand in einem Falle von partieller Sehnervenatrophie bei Tumor hypophyseos cerebri beiderseits Drusen in den Papillen als konglomerierte kuglige Gebilde. Ein Zusammenhang mit der Glaslamelle der Chorioidea liess sich nicht erweisen. Der Patient war ein 63jähriger Mann. 
A. Nieden $(21,1889)$ bringt in einer gründlichen Arbeit 6 neue Fälle und ansserdem eine Beschreibung und Abbildung des späteren Befundes in seinem früher $(6,1878)$ veröffentlichten Falle. Die damals gruppenförmig den Sehnervenring einfassenden, noch nicht zusammenhängenden Wucherungen bildeten nun einen bis anf eine kleine Lücke wallartig den ganzen Optikus begrenzenden Ring, der bis auf 4,5 mm hoch in seinen höchsten Erhebungen in den Glaskörperraum hineinragte. (Fig. 17, Taf. XXXIII.)

Die neuen Falle waren folgende:

1. Bei einem 29jährigen Manne erschien die Oberfläche des rechten Sehnervenquerschnittes wie von einzelnen scharf begrenzten Facetten von Stecknadelkopf- bis Hirsekorngrôsse eingenommen, die im Zentrum der Papille am zahlreichsten vortraten, neben und unter den Zentralgefầssen gelagert schienen, und einen bläulich-grauen Farbenton, dem bei den grösseren Plaques beim Herïbergleiten des Lichtstrahles ein glitzerndes Aussehen beigemiseht war, zeigten. Im Laufe der folgenden Jahre wurde ein fortschreitendes Wachstum des Gebildes, sowohl nach der Breite als Höhe, konstatiert. Zeitweise Exacerbationen im Wachstum und Abnahme der zentralen Sehschärfe wurden beobachtet, denen bald dann wieder zeitweiser scheinbarer Stillstand des Wachstums und Besserung des Sehvermögens folgten. Verfasser meint, dass diese Schwankungen des Sehvermögens dureh zeitweise Kompression der Sehnervenfassern durch die wachsenden Drusen und nachfolgende Adaptation der Fasern an den Druck verursacht waren. Im Laufe der Jahre breitete sich die Drusenbildung fast über die ganze Papillenfläche aus und drängte sich bis zu einer Höhe von $4 \mathrm{~mm}(+12 D)$ in den Glaskörperraum hervor, indem sie eine vielfach höckerige, in unzähligen kleinen Reflexbildern strahlende Erhebung darstellte. Die Gefässe wurden vielfach seitlich verschoben und überlagert, aber nirgends komprimiert.

Die zentrale Sehsehärfe, die anfangs zwischen ${ }^{1 / 3}$ und ${ }^{2 / 3}$ schwankte, war bei der letzten Untersuchung $3 / 5$. Mit Ausnahme einer leichten konzentrischen Einengung der Grenzlinie für Blau war das Gesichtsfeld normal.

Im linken Auge, dessen Sehvermögen immer ganz normal war, wurde zuletzt beginnende Drusenbildung am Rande der Sehnervenscheibe beobachtet. Beide Augen waren übrigens ganz normal.

2. (Fig. 18, Taf. XXXIII.) Auf beiden Papillen eines 28jährigen Mannes fand sich eine Drusenentwicklung, die den zentralen Teil der Papille einnahmen und die Ursprungsstelle der Gefässe verdeckte. Die Sehnervengrenze lag in der grösseren Ausdehnung frei. Die höchste Erhebung des Gebildes lag ungefähr $4,6 \mathrm{~mm}(+14 D)$ über dem Augengrund am linken Auge, minder hoch am rechten Ange.

o. d. $s=1$. Gesichtsfeld mit Ausnahme einer geringen konzentrischen Einengung der Grenze für Blau normal.

o. s. Amblyopie seit frühester Erinnerung. Strabismus divergens. $s=$ Finger in $3 \mathrm{~m}$, Gesichtsfeld konzentrisch eingeengt.

Die übrige Untersuchung gab normale Verhältnisse:

3.-5. Drei Falle boten das Bild der randständigen Drusen dar; 
meist waren zwei bis drei einzelne Plaques, besonders an der inneren Seite der Sehnervenscheibe, aufgekeimt. Es waren Fälle, in welchen weiter keine pathologischen Anomalien des Augenhintergrundes vorhanden waren. Bei zwei derselben hatten schwere Schädelverletzungen stattgefunden, die in starker Quetschung der einen Schädelhälfte bestanden, in einem Falle mit einem bleibenden Enophthalmus kompliziert. Die Drusen fanden sich nur im Optikus der verletzten Kopfhälfte, und deren Entwicklung begann - jedenfalls in einem Falle - erst mehrere Monate nach dem stattgehabten Trauma.

6. Bei einem 24jährigen, hereditär psychisch belasteten und seit 2 Jahren an den Erscheinungen schwerster Neurasthenie und nervöser Asthenopie leidenden Mann wurde Drusenentwicklung am Sehnervenrande bei sonst normalem Spiegelbefunde und gleicher Sehschärfe gefunden.

Die Anschaunng, dass die Sehnervendrusen Produkte der Glaslamelle der Chorioidea wären, gegen welche, wie erwähnt, nur Oeller vergebens protestiert hatte, wurde endlich durch Arbeiten von Hirschberg und Cirincione $(22,1891)$ and von Gurwitsch $(23,1891)$ entschieden umgestürzt.

Der von Hirsehberg klinisch und von Cirincione mikroskopisch beschriebene Fall war folgender: Bei einem 59jährigen Fräulein wurde das linke Auge wegen Aderhautsarkoms enucleiert. Am rechten Ange wurde als einzige Anomalie eine Veränderung des Sehnerveneintrittes konstatiert (Fig. 10, Taf. XXXIII), die Substanz des letzteren war rötlich, die Begrenzung unregelmässig dureh knollige glänzende Bildungen, die mehr oder weniger tief im Gewebe lagen und über dem scheinbaren Rand des Sehnerven in die benachbarte Netæhant hinübergriffen, während der mittlere Teil der Sehnervenscheibe mehr frei geblieben war; es waren offenbar körperliche, kuglige Bildungen mit glänzender Umrandung. Auch in der angrenzenden Netzhantzone war ein und der andere glitzernde Punkt sichtbar. Während der Beobachtungsdauer (ungefähr 3 Monate) schienen diese Drusen nicht zuzunehmen. Das Auge hatte normale Sehkraft und normales Gesichtsfeld.

Die anatomische Untersuchung des enucleierten Augapfels zeigte, ausser dem melanotischen Spindelzellensarkom der Aderhaut, ein im Innern der Papille ringförmig um die Zentralgefässe abgelagertes Konkrement. Die sehr genaue histologische und mikrochemische Untersuchung dieses Konkrementes ergab, dass es sich um eine Ablagerung von hyalinen Massen handelte, die zum Teil sekundär verkalkt waren. Sie bestanden aus amorphen organischen Körpern nicht zelliger Natur, die keine Eiweiss-, Fett- oder Leimreaktion gaben und deren indifferentes Verhalten gegenüber den gebräuchlichen Lösungsmitteln sie dem Elastin am ähnlichsten erseheinen liess. Es fanden sich keine Zeichen eines entzündlichen Prozesses, sondern ein partieller Nervenfaserschwund, der auf einem einfachen Degenerationsvorgang als Folgeerseheinung des durch das Wachstum des Gebildes bedingten Druckes bezogen werden musste.

An die Beschreibung dieses Falles knüpft Hirschberg einige Bemerkungen über das Vorkommen und die Bedeatung der Drusen im Sehnervenkopf an. Er erwähnt, dass sie relativ häufig bei Retinitis 
pigm. vorkommen, ohne, wie es scheint, zur Sehstörung beizutragen. In sonst gesunden Augen sind sie nicht allzuselten, im stärkeren Grade doch sehr selten; die Sehkraft bleibt, auch bei diesem Grade, normal. Die Veränderung kommt wohl nicht bei Kindern, sondern gewöhnlich bei Erwachsenen, keineswegs nur bei Greisen vor.

Zwei Fälle wurden mitgeteilt:

1. (Fig: 7, Taf. XXXIII.) Bei einem 25jährigen Herrn mit normaler Sehfunktion fanden sich knollige Drusen am Rande des rechten Sebnerven. Er war unmittelbar vorher anderswo wegen Sehnervenentzündung 9 Wochen behandelt worden.

2. Bei einer 40jährigen Dame fand sich Drusenbildung im Sehnervenkopf beiderseits, eine Staunngspapille vortäuschend. Links war auch ein kleiner glitzernder Punkt getrennt vom Sehnerven in der Netzhaut zu sehen. Die Sehkraft war gut. Die Patientin hatte vor 14. Tagen eine Lähmung des rechten Gesichtsnerven erlitten, die noch deutlich hervortrat.

M. Gurwitseh $(23,1891)$ teilte ungefähr gleichzeitig die mikroskopische Untersuchung der Augen eines 34jährigen Nephritikers mit. Beide Augen zeigten leichte auf Nephritis beruhende Veränderungen in der Retina; ausserdem fanden sich in einem Auge geschichtete Konkretionen in der Papille hauptsächlich in der Nähe der Lamina cribrosa. Verfasser meinte mit Sicherheit behaupten zu können, dass sie nicht Drusen der Lamina vitrea chorioidea vorstellten, und gab an, dass sie vollkommen den Gebilden, die von v. Recklinghausen als hyaline bezeichnet sind, entsprachen.

Nach diesen, für die pathologische Anatomie der Drusen sehr bedentungsvollen Arbeiten folgt in den späteren Jabren eine Reihe von Mitteilungen, die wesentlich nur das kasuistische Material vergrössern.

O. Purtscher (24, 1891) [Fig. 14, Taf. XXXIII] fand bei einer 35jährigen Patientin starke Drusenbildung in beiden Papillen; in allen Teilen der Sehnervenscheibe fanden sich massenhafte, zum Teil stark hervorragende, hellglänzende Einlagerungen, deren scheinbare Grösse von Stecknadelkopf- bis Erbsengrösse und darüber schwankte. Die Gefässe waren normal. S. 0. d. $6 / 9$, 0. s. Finger in $0,3 \mathrm{~m}$. (Patientin klagte ubber Schlechtsehen des linken Auges seit drei Wochen.) Peripheres Gesichtsfeld frei.

G. E. de Schweinitz $(25,1892)$ hatte Gelegenheit in einem Falle, in dem Drusenbildung ophthalmoskopisch beobachtet war, später die mikroskopische Untersuchung vorzunehmen. Der 45 jährige Patient war geistesschwach, wahrscheinlich wegen Trunkes, und war vor 20 Jahren in den Kopf geschossen. S. o. s. Finger in $3^{\prime}, 0$. d. Finger in $6^{\prime}$. Die Papillen waren leicht prominent, und die aus glänzenden Partikelchen zusammengesetzten globulären Massen bildeten einen Ring am Rande derselben. Die anatomischen Veränderungen bestanden in hyalinen Bildungen in der Papille von der Lamina cribrosa, Atrophie des Optikus und Verdickung der Zentralarterie.

A. Terson $(26,1892)$ besehrieb das mikroskopische Bild eines Falles von Glaukom, bei dem die hyalinen Massen im atrophischen und degene- 
rierten Gewebe in der Wand der Excavation eingelagert waren; sie bestanden aus einer amorphen, konzentrisch geschichteten Masse und waren ohne Kapsel im Gewebe eingebettet.

Peters $(27,1893)$ beschrieb einen Fall von Drusenbildung am Sehnerven.

G. E. de Schweinitz $(28,1894)$ fand in zwei Fällen ähnliche Gebilde in der Macula.

A. Sachsalber $(29,1895)$ [Fig. 8 u. 9, Taf. XXXIII] teilte die klinisehe und mikroskopische Untersuchung eines Falles von Drusen in beiden Papillen mit. Die 42jährige Patientin, die später an Carcinoma ventriculi starb, hatte am rechten Ange $\left.S^{6}\right|_{9}$, am linken $\left.S^{6}\right|_{6}(?)$, und beiderseits geringe konzentrische Gesichtsfeldeinengung. An beiden Augen fanden sich bis zu $3-4 D$ prominierende grauweisse Gebilde am Papillenrande; sie waren im Zentrum heller mit einem dunkleren Saum und zeigten an ihrer Oberfläche hier und da glitzernde Punkte; die Papillen waren etwas gerötet, der Augengrund im übrigen normal. Anatomiseh fanden sich in beiden Sehnerven - zum Teil anch hinter der Lamina cribrosa - Ablagerungen einer Albumenreaktion gebender Substanz, welche die von v. Recklinghausen geforderten Eigenschaften des Hyalins darbot; in späteren Stadien zeigten sie sekundäre Veränderungen und Verkalkungen. Amyloidreaktion fiel ganz negativ aus. Ausserdem fanden sich in den Sehnerven die Zeichen von chronischer interstitieller Neuritis.

Verfasser nimmt an, dass die Drusen immer auf konkreten entzündlichen Vorgängen beruhen.

H. Gifford $(30,1895)$ beobachtete bei einem 11jahrigen Mädchen eine grosse, ans kugligen hyalinen Körpern bestehende Wucherung, die durch eine leichte Einschnürung in zwei Teile geteilt war. Sie deckte ganz die Papille und die umgebende Retina und hob sich ungefahr $3 \mathrm{~mm}$ $(9 D)$ über den Fundus. Die oberen Arterien waren in weisse Bänder verwandelt, und es fanden sich im Glaskörper frische Hämorrhagien. Die Sehkraft war seit 2 Jahren stark vermindert.

Bei einem 45jährigen Mann mit ausgeprägten Drusen in beiden Papillen entwickelte sich nach einem Jahre Brightsche Krankheit, woran er das folgende Jahr starb.

Heyl $(31,1895)$ fand bei einer 49 jährigen Frau beiderseits weissliche Infiltration und Pigmentveränderungen im Augengrund bei gleichzeitigem Vorhandensein typischer Drusenbildung in der Papille und ähnlicher Gebilde in der Macula und nach innen von der Papille.

E. S. Thomson $(32,1898)$ fand bei einer 29 jăhrigen Frau sekundäre Atrophie des Optikus nach Neuritis, verbunden mit'Ablagernng. kolloider Massen auf der Papille als zahlreiche kleine wachsähnliche Kügelchen.

A. Nieden $(33,1900)$ beschreibt das gegenwärtige ophthalmoskopische Bild in seinem zweimal früher $(6,1878 ; 21,1889)$ referierten Falle. Die Drusen ragen nun bis zu 5 und $6 \mathrm{~mm}$ im Glaskörper hervor, drängen sich als gestielte, blumenkohlartige glitzernde Auswüchse zwischen den Gefässen hervor, ohne dieselben zu drücken; einige zeigen bei Augenbewegungen schlotternde Mitbewegungen. Sie finden sich wie früher nur 
in der Randzone der Papillen. Die ältesten wie die jüngsten bieten genau dasselbe Bild dar, es finden sich keine Zeichen von Kalk- oder Cholestearinablagerungen.

F. Mendel $(34,1900)$ fand bei einer 24 jährigen, an Hysterie und Bleichsucht leidenden Patientin Drusen in den Papillen, deren Grenzen verwasehen waren; die Drusen fanden sich rechts am nasalen Rande, links mehr innerhalb der Grenze der Papille. S. o. u. $\left.{ }^{5}\right|_{4}$, Gesichtsfeld normal.

A. Stanford Morton $(35,1903)$ beobachtete zwei Fäle:

1. (Fig. 16, Taf. XXXIII.) 24jähriger Mann mit vorgeschrittener Retinitis pigmentosa. In beiden Augen Drusen an den Papillen, die sich während drei Jahren nicht veränderten, während die Sehschärfe von $3 / 60$ o. d., $6 / 24$ o. s. bis an $<\left.1\right|_{60}$ o. d., $\left.{ }^{1}\right|_{60}$ o. s. abnahm und das Gesichtsfeld bis an $5^{\circ}$ eingeengt wurde. Die Eltern waren Geschwisterkinder.

2. 20jährige Frau. S. o. d. ${ }^{6} / 9$, 0. s. ${ }^{6 / 5}$. Die Gesichtsfelder zeigten bei normalen Grenzen ein oder zwei kleine Skotome. Ophthalmoskopisch fand sich distinkte Blässe der Papillen und Drusen in beiden. Die Eltern waren verwandt.

Herbert Parsons $(35,1903)$ hat einen Fall von Papillendrusen mikroskopisch untersucht und fasst die Drusen als umgebildete Exsudate auf.

Hanke $(36,1904)$ fand bei einem 9jährigen hypermetropischen Mädchen nach innen oben von der Papille einen um $6 D$ prominierenden, scharf abgegrenzten, über $2 P D$ im Durchmesser grossen Tumor von feinhöckeriger, perlmutter glänzender, stellenweise opalisierender Oberfläche. Visus $6 F_{5}$. Er bezeichnete den Tumor als eine hyaline Excrescenz der Chorioidea.

Streiff (37, 1904) hat 5 Fälle beobachtet:

1. 17jähriges Mädchen, 3Jahre zuvor wurde beiderseitige Stauungspapille nebst Drusen an der linken Papille gefunden. Nun Drusen mässigen Grades in beiden Papillen. $\$$. 0. u. $\left.{ }^{6}\right|_{8}$, Gesichtsfeld unregelmässig eingeengt.

2. Eine 32 jährige Frau zeigte ausgesprochene Staurngspapille an beiden Augen, namentlich am rechten. Nach 10 Monaten waren beide Papillen flacher, in der linken fanden sich Drusen in geringem Grade. S. o. a. ${ }^{6} / 12$, 0. s. ${ }^{6} / 18$,

3. Bei einem 20jährigen Mann waren die Papillen etwas verfärbt, unscharf begrenzt, von granem Hof umgeben und leicht (ungefähr $2 D$ ) geschwollen. (Abgelaufene Stauungspapille?) In der linken Papille fand sich Drusenbildung am oberen und unteren Rand. Sehnerven und Gesichtsfeld beiderseits normal.

4. Bei einem 26jăhrigen Mann wurde geringe Drusenbildung in beiden Papillen, die prominent und unscharf begrenzt waren, gefunden. (Abgelanfene Stauungspapille?) S. o. u. $6 / 6^{\circ}$

5. Bei einer 55jährigen Frau waren beide Papillen grauweiss, unscharf begrenzt und zeigten Drusenbildung mässigen Grades. S. o. d. $\left.{ }^{6}\right]_{6}, 0.5$. $\left.{ }_{6}^{6}\right|_{8}$. Gesichtsfeld beiderseits unregelmässig eingeengt. (Partielle Atrophie nach Stauungspapille oder Neuritis.) 
Demaria $(38,1904)$ fand bei Untersuchung des wegen Glankoma absolutum enucleierten Bulbus einer 54jahhrigen Patientin die Druckexcavation mit einer Masse konglomerierter "Corpora amylacea" ausgefüllt. Dieselben färbten sich mit Jodtinktur gelb, durch Nachbehandlung mit dünner Schwefelsäure blänlich.

Cirincione $(39,1904)$ teilte zwei Falle mit, der eine war mikroskopisch untersucht. Er fasst die Drusen als Produkte einer langsamen und andauernden Ablagerung hyaliner Substanz zwischen den Maschen des Stützgewebes auf, wodureh die Sehnervenfasern ohne Beeinträchtigung ihrer Funktion oder Struktur auseinandergedrängt werden.

Rabitsch $(40,1905)$ fand bei einer 24jährigen Frau, die seit frühester Jugend schlecht gesehen hatte, starke Drusenbildung in beiden Papillen, namentlich in ihrer Peripherie; die Mehrzahl der Drusen zeigten einen deutlichen kugligen Charakter, einige sahen dadurch, dass der Lichtreflex beständig den Rand einnahm, fast napfförmig aus. Die Sehschärfe war o. d. 0,2 , 0. s. 0,6, das Gesichtsfeld beiderseits defekt (Taf. XXXIII, Fig. 15).

Ausserdem teilte er den mikroskopischen Befund von Drusen im Sehnervenkopf des normalen Bulbus eines 70 jährigen Mannes mit, bei dem intra vitam weder subjektiv noch objektiv Abnormes bestanden hatte. Es fanden sich hier gar keine Zeichen von Entzündung in den Papillen. Schuster $(41,1906)$ (Taf. XXXIII, Fig. 13) fand bei einem 37jährigen Mann starke Drusenentwicklung in beiden Papillen; die ganze Papillenmasse zeigte sich von einem Mosaik glitzernder, teils kugliger, teils mehr scholliger Konkremente zusammengesetzt, die eine unregelmässige Begrenzung sowohl gegen die Hyaloidea wie nach dem Papillenrand hin verursachten. Die Papillen waren im ganzen ungefähr $2 D$ geschwollen; die Gefässe waren eine Strecke in die Retina hinaus in bläulichgrauen Scheiden eingescheidet. Die linke Linse zeigte eine offenbar kongenitale Trübung in Form von supranueleär zentral gelegenen, bläulichen, annähernd in Sternform angeordneten Punkten. Im übrigen waren die Augen normal; Visus und Gesichtsfeld waren auf beiden Augen normal.

Harman $(42,1906)$ fand bei einem Patienten die linke Papille anscheinend mit hyalinem Material infiltriert; gleichzeitig fanden sich in der Macula einige kleine glitzernde Fleckehen.

Bonh off $(43,1906)$ teilte einen Fall von Psendoneuritis mit Drusen in der Papille mit. Der 18jährige Patient, der beiderseits normales Sehvermögen bei H. 4,00 hatte, litt ausserdem an Flimmerskotom. Die Drusen zeigten sich als wenige rundliche, hellglänzende, gelblich durchschimmernde Flecken in der Nachbarschaft der Hauptvenen. Die Papillen waren dabei undeutlich begrenzt, graurötlich, nicht prominent. Die Gefässe waren normal.

Lauber $(44,1907)$ teilte 5 Fälle mit. Der eine war derselbe, den Rabitsch (40, 1905) vor zwei Jahren mitgeteilt hatte; der ophthalmoskopische und funktionelle Befund war unverändert geblieben. Die übrigen vier Fälle waren folgende:

1. Bei einem 15 jährigen Mädchen fanden sich in beiden Papillen, 
ihre normale Struktur vollständig verdeckend, grössere Mengen einer leicht durchscheinenden, grauweissen, krystallinisch aussehenden Substanz, die beiderseits eine Refraktionsdifferenz von $2 D$ gegenüber der Netzhaut aufwies. $S=1$ o. u.

2.-4. Eine 67 jährige Fran und ihre zwei (42 jährige nnd 41 jährige) Töchter. Bei allen drei war $S$ o. u. $=1$. Bei der Mutter fanden sich Drusen in den Randpartien beider Papillen, bei der ältesten Tochter reichliche Einlagerung von Drusen in den Papillen, den Ursprung der Gefässe verdeckend, bei der jüngsten Schwester im linken Auge ein ähnlicher Befund wie bei der älteren, während im rechten Auge nur geringe Andeutung von Drusenbildung in der temporalen Papillenhälfte vorhanden war.

Wenn man versuchen will, auf Grundlage der hier beschriebenen und der aus der Literatur gesammelten Fälle sich ein allgemeingültiges Bild vom Auftreten des betreffenden Leidens $z u$ bilden, dann ist natürlich zuerst zu untersuchen, ob alle die Fälle sich unter den gleichen Krankheitsbegriff hineinbringen lassen, ob es anzunehmen ist, dass der gleiche pathologische Prozess sich in allen abspielte.

Ich glaube, dass diese Frage in bezug auf den weitaus grössten Teil der Fälle sich bejahend beantworten lässt. In den mikroskopisch untersuchten Fällen ist die Beschreibung des Befundes sehr gleichartig; es dreht sich immer um hyaline, rundliche, in grösseren oder kleineren Mengen im Papillengewebe eingelagerte, konglomerierte Körnchen; die Verbindung mit der Lamina vitrea chorioideae, die Iwanoff(2) nachzuweisen meinte, ist wohl als auf zufälligen Verhältnissen beruhend aufzufassen, die keine Grenze zwischen seinen und den übrigen Fällen ziehen. Nur ein Fall, nämlich Demarias(38), weist einen wesentlichen Unterschied von den übrigen auf, da die Amyloidreaktion, die sonst immer negativ ausfiel, hier ein positives Resultat gab; in allen sonstigen Beziehungen ist der Befund aber dem in den übrigen Fällen ähnlich, und der Fall ist wohl jedenfalls als mit den andern sehr nahe verwandt anzusehen.

Die klinischen Befunde lassen sich ganz zwanglos auf Grundlage der beschriebenen pathologisch-anatomischen Bilder erklären; dass es wirklich der gleiche Prozess ist, um den es sich sowohl in den anatomisch als in den ophthalmoskopisch untersuchten Fällen handelt, wird ferner bekräftet durch Hirschbergs und Cirinciones Fall (22), bei dem das Krankheitsbild im einen Auge anatomisch, im andern Auge des gleichen Patienten klinisch nachgewiesen wurde, and namentlich durch de Schweinitz' (25) und Sachsalbers (29) Fälle, in denen die Drusen im Sehnervenkopf sowohl ophthalmoskopisch als auch mikroskopisch konstatiert wurden. 
Das sehr charakteristische klinische Bild wird in den verschiedenen Fällen ganz übereinstimmend beschrieben. Die Verschiedenheiten, die sich finden, lassen sich leicht als Verschiedenheiten der Entwicklungsstufen und Lagerung im Papillengewebe erklären. Nur in einem Falle, nämlich Remaks (17), erweckt die Beschreibung einigen Zweifel, ob er mit den übrigen zusammengehöre. Das tumorartige Gebilde, das Remak an der Stelle der Papille fand, bestand nach seiner Beschreibung aus fünf oder sechs zusammenhängenden Teilen, die doch so gross waren, dass man sie nicht als einzelne Drusen auffassen kann, und für die Anschauung, dass jeder derselben aus zusammengehäuften Drusen bestände, gibt weder die Beschreibung noch das beigefügte Bild einigen Anhalt. Wenn auch die Diagnose Drusen vielleicht am wahrscheinlichsten ist, lässt es sich doch auch denken, dass die Excrescenz aus Bindegewebe bestände, und dass der Fall also mit Fällen von Retinitis proliferans verwandt wäre.

Unter den übrigen Fällen finden sich einige, wo die Drusen so wenig entwickelt, und andere, wo das Bild so kurz beschrieben ist, dass man sie nur mit Vorbehalt mitzählen darf, und hierzu kommen für mich die Fälle, die mir nur durch kurze Referate bekannt sind. Doch bleibt ein genügend grosses Material charakteristischer und gegenseitig übereinstimmender Fälle, um einen Eindruck der typischen und gemeinschaftlichen Eigenschaften derselben zu geben.

Die Verschiedenheiten, die sich zwischen den einzelnen Fällen finden, sind wesentlich von drei Arten:

Erstens zeigt sich ein grosser Unterschied in der Menge der Drusen und der Grösse der Konglomerate, aber dieser Unterschied ist ganz natürlich dadurch zu erklären, dass die Drusen in den verschiedenen Fällen auf ganz verschiedenen Entwicklungsstufen beobachtet sind. Unter den beschriebenen Fällen findet man alle denkbaren Übergänge, von den leichtesten bis zu den schwersten Formen, und in mehreren Fällen ist es möglich gewesen, die Entwicklung der Drusenbildung durch einen längeren Zeitraum, bis zu mehreren Jahren, zu verfolgen, und dadurch den Übergang von weniger bis weiter vorgeschrittenem Stadium zu konstatieren.

Einen ferneren Unterschied bildet die Lagerung der Drusen in der Papille, indem sie in den meisten Fällen im Papillengewebe, tiefer oder oberflächlicher, eingelagert sind, während sie in selteneren Fällen vor der Papille als frei prominierende Konglomerate im Glaskörper zu sehen sind. Dieser Unterschied lässt sich ungezwungen erklären, wenn man annimmt, dass die Drusenmasse in den letzteren Fällen die Oberfläche 
der Papille durchbrochen hat, und dass die Bildung neuer Drusen in der Papille beständig die älteren gegen das Innere des Auges verschiebt. Auch hier sieht man Übergangsformen, da in mehreren Fällen, wo die Drusen hauptsächlich im Papillengewebe eingelagert waren, einzelne Teile derselben in dem Glaskörper prominierten. Ausserdem zeigen die einzelnen Elemente der Konglomerate sehr grosse Übereinstimmung in ihrem Aussehen, sei es dass sie frei im Glaskörper oder in der Papille eingebettet liegen.

Von grösserer Bedeutung ist die Frage, ob es als ein wesentlicher Unterschied anzusehen sei, dass die Drusen sich bisweilen in gesunden, oder jedenfalls anscheinend gesunden Augen entwickeln, während sie in andern Fällen als Komplikation eines primären Leidens auftreten, mit andern Worten, ob die Fälle in zwei Gruppen, den primären und den sekundären, zu teilen seien. Es scheint mir doch kein Grund vorhanden, eine solche Einteilung vorzunehmen. Die Drusen treten unter beiden Umständen auf ganz dieselbe Weise auf, sie weisen ganz dieselben Merkmale auf, und können denselben Entwicklungsgrad erreichen. Wenn die Papille der Sitz objektiver krankhafter Veränderungen ist, dann wird das Krankheitsbild natïrlich in toto etwas anders, die für die Drusen eigentümlichen Kennzeichen ändern sich aber nicht; ausserdem sind die Drusen offenbar nicht besonders fest abhängig von dem Leiden, bei welchem sie auftreten; sie zeigen sich immer als eine seltene und, wie es scheint, zufällige Komplikation, und die Menge, in der sie gebildet werden, scheint ganz unabhängig vom Grad und Charakter des betreffenden Leidens.

Bei der Beschreibung des Krankheitsbildes ist es deshalb natïrlich, sämtliche Fälle auf einmal zu behandeln und nur die verschiedenen Entwicklungsstadien als Grundlage der Darstellung zu verwenden, sowie auch Hirschberg(22) getan hat.

In ihrem ersten Anfange zeigen sich die Drusen als feine, helle oder glänzende Punkte oder Fleckchen im Papillengewebe, gewöhnlich in der Nähe des Randes, bisweilen ein wenig ausserhalb dieses (also in der Retina); in diesem Stadium ist die Diagnose indessen als unsicher anzusehen, weil es sich sehr wohl denken lässt, dass solche kleinen Punkte oder Fleckchen ohne bestimmte Form oder Merkmale, namentlich wenn sie sich in pathologisch veränderten Papillen zeigen, anderer Natur sein können, sowie Ablagerungen von krystallinischer oder amorpher Stoffe anderer Art (Kalk, Cholestearin, Fett). Sicher wird die Diagnose, wenn sich Gelegenheit bietet, die Entwicklung der kleinen Pünktchen zu charakte- 
ristischen Drusen zu verfolgen, sowie es auch in einzelnen Fällen geschehen ist. Mit grösserer oder kleinerer Wahrscheinlichkeit lässt die Diagnose in diesem Stadium sich stellen, wenn sich im andern Auge des Patienten charakteristische Papillendrusen finden [mein Fall I, Nieden(21) Fall I], oder wenn dieses, sowie in Anckes Fällen (16), bei den Geschwistern des Patienten der Fall ist. In solchen Fällen wie mein Fall $V$ lässt es sich dagegen nicht entscheiden, ob die kleinen hellen Fleckchen, deren Aussehen nichts Charakteristisches darbietet, ein solches Anfangsstadium repräsentieren.

Erst in einem etwas weiter vorgerïckten Stadium werden die Drusen als solche erkennbar, sich präsentierend als rundliche, gruppierte oder konglomerierte Gebilde von einer stark lichtreflektierenden, graulichweissen oder bläulichweissen Farbe. Sie treten mit überwiegender Häufigkeit in den Randteilen der Papille auf und können abgegrenzte Konglomerate bilden oder mehr zerstreut liegen. Diesem Entwicklungsstadium gehören zahlreiche von den beschriebenen Fällen an, z. B. Hirschbergs (22) Fall III, Sachsalbers(29) und mein Fall IV (Taf. XXXII, Fig. 5-6 und Taf. XXXIII, Fig. 7-9).

Die weitere Entwicklung kann in zwei Richtungen gehen. Die Drusen können fortwährend im Papillengewebe eingelagert bleiben, sich in diesem ausbreitend, so dass die Papille allmählich in grösserer Ausdehnung mit ihnen gefullt wird [Stood (9), Hirschberg(22), mein Fall III; Taf. XXXII, Fig. 3-4 und Taf. XXXIIT, Fig. 10-12]. Die Drusen lagern sich dann in verschiedenem Niveau, zum Teil einander verdeckend; die oberflächlichsten bilden unregelmässige knollige Vorsprünge an der Oberfläche der Papille. Zuletzt wird die ganze Papille mit Drusen infiltriert, verliert dadurch ihr natürliches Aussehen und scheint fast nur aus dicht zusammengeballten Drusen zu bestehen; durch das Wachstum der Drusenmasse wird sie vergrössert, prominent, und kann dadurch eine Stauungspapille vortäuschen. Die Ränder werden unregelmässig, da die Drusen auch hier gegen den benachbarten Augengrund halbkreisförmige oder unregelmässige Vorsprünge machen. Die Papille bekommt im ganzen einen eigentümlichen bläulich- oder gränlichweissen Farbenton. Die Gefässe verlaufen über oder um die vorspringenden Partien, und werden dadurch bisweilen zu einem geschlängelten Verlauf gezwungen; dagegen zeigen sie keine Veränderungen in Farbe oder Kaliber. In einem solchen stark vorgeschrittenen Stadium sind mehrere Fälle beschrieben [Purtscher (24), Rabitsch (40), Schuster (41), mein Fall I; Taf. XXXII, Fig. 1 und Taf. XXXILI, Fig. 13-15]. 
In einigen Fällen bleibt dagegen ein grosser oder der grösste Teil der Papille fortwährend von Drusen verschont, indem dieselben sich nur in einem begrenzten Teil der Papille bilden, und von hier aus über deren Oberfläche in den Glaskörper hervordringen und tumorartige Konglomerate bilden. Das ophthalmoskopische Bild ist in solchen Fällen überaus prachtroll. Die aus Massen von kleinen glänzenden Kügelchen zusammengesetzten Excrescenzen glitzern und schimmern in der ophthalmoskopischen Beleuchtung und heben sich hoch über das Niveau des Augengrundes, grössere oder kleinere Partien der natürlich aussehenden Papille verdeckend. Die Höhe, zu welcher die Drusenmasse sich im Glaskörper erhebt, kann sehr bedeutend sein. Nieden notiert in seinen Fällen Erbebungen bis zu $4-4,6 \mathrm{~nm}$ iuber dem Augengrund (12,0-14,0 Dioptr. Refraktionsunterschied). Gifford fand in seinem Fall $3 \mathrm{~mm}$ (9 Dioptr.). Die Gefässe werden bisweilen von den Excrescenzen emporgehoben oder sie durchbohren dieselben; in der Regel aber behalten sie ihren natürlichen Verlauf und werden also von den Drusenmassen überdeckt. Der benachbarte Glaskörper ist immer vollständig klar, so dass selbst die kleinsten Details ganz scharf zu sehen sind. Die einzelnen Elemente der Drusenkonglomerate scheinen weit regelmässiger und gleichartiger in Form, Farbe und Grösse zu sein als die im Papillengewebe infiltrierten Drusen, vermutlich weil der Glaskörper ihrem Wachstum geringeren Widerstand leistet.

Fälle dieser Art sind seltener als die der vorigen Gruppe. Nie den beschreibt 3 Fälle dieser Art, Gifford 1, Morton 1 und hierzu kommt mein Fall II (Taf. XXXII, Fig. 2, Taf. XXXIII, Fig. 16-18). Auch Remaks Fall ist mitzurechnen, wenn es sich hier um Drusen handelt, was mir, wie gesagt, etwas unsicher vorkommt.

Obwohl die Papille in der Regel der Sitz der Drusen ist, kommt es doch auch vor, dass sie sich ausserhalb derselben in der Retina entwickeln. Nicht allzu selten sind in den beschriebenen Fällen vereinzelte kleine Drusen ausserhalb des Papillenrandes beobachtet; aber auch grosse Konglomerate in grösserer Entfernung von der Papille, zum Teil in der Macula, werden beschrieben [Lawson(10), de Schweinitz (28), Gifford (30), Heyl (31), Hanke (36)]. Leider sind alle diese Arbeiten mir nur durch kurze Referate bekannt, und ich getraue mich deshalb nicht zu entscheiden, ob diese Bildungen wirklich von ganz derselben Art wie die Papillendrusen seien.

Die einzelnen Elemente der Konglomerate, die einzelnen "Drusen“, haben ein sehr charakteristisches Aussehen und verhalten sich in den 
verschiedenen Fällen sehr gleichartig. Sie präsentieren sich immer als stark lichtreflektierende Gebilde von einer eigentümlich glänzenden, bläulich- oder graulichweissen Farbe, die gewöhnlich als perlmutterartig bezeichnet wird, die aber eher - durch ihren gleichartigen halbdurchscheinenden Charakter - mit der Farbe dünnen, leicht bläulichen Milchglases zu vergleichen wäre. Die Form ist in der Regel ganz oder beinahe kuglig, was am besten an den Konturen zu sehen ist, die fast immer cirkulär sind, sei es, dass die Drusen auf der Papillenfläche als kreisförmige Flecken zu sehen sind, oder dass sie von den Rändern der Papille oder eines Konglomerats prominieren, halbzirkelförmige Vorsprünge gegen den hinterliegenden Augengrund zeichnend; meist regelmässig ist die Form der Drusen, wenn sie den frei im Glaskörper prominierenden Konglomeraten angehören; die im Papillengewebe infiltrierten Drusen zeigen recht oft mehr unregelmässige Formen, länglich oder polygonal mit abgerundeten Ecken.

Die Grösse der Drusen variiert nicht wenig; sie können alle Übergänge von ganz kleinen bis grossen zeigen; der Durchmesser der grössten übertrifft gewöhnlich nicht sehr die Breite der Hauptvenen. Die grössten findet man. unter den im Papillengewebe infiltrierten Drusen; in den Konglomeraten im Glaskörper sind sie im ganzen kleiner und von mehr gleichartiger Grösse; dies ist sehr deutlich, wenn man die Bilder der Niedenschen Fälle und meines Falles II mit den übrigen Abbildungen vergleicht.

Die Weise, auf welche die Drusen bei der ophthalmoskopischen Untersuchung das Licht reflektieren, ist recht auffällig. Es liesse sich a priori erwarten, dass solche kugelförmigen Körperchen einen zentralen Reflex geben würden; dies scheint aber nicht der Fall zu sein. Die ganze nach vorn gegen den Beobachter gerichtete Fläche zeigt sich gleichmässig lichtreflektierend, wogegen die Peripherie der Drusen oft stärker glänzt, so dass sie von einem helleren Saume umgeben scheinen; sie gewinnen dadurch ein Aussehen, das beim ersten Anblick leicht eine falsche Vorstellung von ihrer Form geben könnte; in verschiedenen Beschreibungen werden sie deshalb als anscheinend knopfförmig, schalenförmig oder schuppenartig bezeichnet, während der Autor sich doch dariber klar ist, dass dieses Aussehen eine Täuschung ist; es ist auch nicht schwierig, durch Beobachtung der Konturen und der parallaktischen Verschiebung die Kugelform zu konstatieren.

Wenn die Drusen tiefer im Gewebe gelagert sind, dann ist ihr 
Aussehen minder charakteristisch, indem es vom überliegenden Gewebe verschleiert wird. Die Konturen zeichnen sich nicht so scharf, die Farbe ist nicht so glänzend, der Randreflex fehlt. Wenn nicht gleichzeitig oberflächliche Drusen vorhanden sind, möchte die Diagnose unter solchen Umständen bisweilen unsicher sein, und es ist wohl auch wahrscheinlich, dass sehr tief dicht auf der Lamina cribrosa gelagerte Drusen sich bisweilen der ophthalmoskopischen Beobachtung ganz entziehen.

Die Entwicklung der Drusen geht immer sehr langsam vor sich, und ihr Wachstum ist nur wahrzunehmen, wenn die Beobachtungsdauer lang ist; selbst bei recht langer Beobachtungsdauer sieht man doch nicht immer die Drusen sich vermehren; Morton(35) konnte in drei Jahren, Hirschberg(22) in drei Monaten und ich (Fall II) in sechs Monaten keine Veränderung der Drusen wahrnehmen; es ist demnach wahrscheinlich, dass die Drusenbildung, wenn sie einen gewissen Grad erreicht hat, einhalten kann. In dem Fall, in dem der Entwicklungsverlauf am genauesten beobachtet wurde [Nieden (21) Fall 1], zeigte das Wachstum der Drusenmassen wechselnde Fortschritte und Stillstand, ohne dass Ursachen davon nachzuweisen waren.

Die Drusen kommen fast immer doppelseitig vor und sind dann in der Regel beiderseits im beinahe gleichen Grade entwickelt, was vielleicht oft davon abhängt, dass sie als Komplikation eines doppelseitigen Leidens auftreten, das beide Augen gleichmässig ergriffen hat. Wenn sie ein einseitiges Leiden komplizieren, dann zeigen sie sich, wie zu erwarten, nur auf der betreffenden Seite. Auch in Fällen, wo ein primäres Leiden nicht nachzuweisen ist, sieht man bisweilen ein einseitiges Auftreten der Drusen; in solchen Fällen können indessen später Drusen im andern Auge auftreten [Nieden(21) Fall 1]; auch in den Fällen, wo grosser Unterschied des Entwicklungsgrades in beiden Augen sich zeigt; ist es wohl anzunehmen, dass der Anfang der Drusenbildung zu verschiedener Zeit stattgefunden hat.

Das Krankheitsbild ist also - vom Anfangsstadium abgesehen - wohl ausgesprochen und leicht kennbar, und die Diagnose wird keine Schwierigkeiten bieten, da es schwerlich eine andere Anomalie gibt, die dasselbe Bild von rundlichen konglomerierten, lichtreflektierenden Gebilden zeigt. Dagegen können die Drusen Anlass zu diagnostischen Schwierigkeiten anderer Art geben, indem sie das 
Aussehen der Papille maskieren und dadurch es schwierig oder unmöglich machen zu beurteilen, ob die Papille in andern Beziehungen normal sei, und speziell, ob eine vorhandene Entfärbung oder Schwellung derselben als nur von den Drusen verursacht aufzufassen sei, oder zugleich als Zeichen von einer florierenden oder abgelaufenen Neuritis oder Stauungspapille.

Wenn einige Verfasser die Möglichkeit erwähnen, Drusen mit Stauungspapillen oder Neuritis zu verwechseln, dann ist es wohl nicht so sehr die Möglichkeit die Drusen zu verkennen, als die Möglichkeit aus dem Aussehen der Papillen auf das gleichzeitige Vorhandensein eines der genannten Leiden zu schliessen und die Drusen als eine sekundäre und verhältnismässig bedeutungslose Erscheinung aufzufassen. Diese Möglichkeit ist in der Tat naheliegend in Anbetracht des sehr auffälligen Aussehens, das die Papille bei starker Drusenbildung darbietet, und das leicht den Gedanken auf eine starke pathologische Veränderung lenkt; in den Zeiten, da die Drusen des Sehnervenkopfes nur durch ganz wenige Mitteilungen vereinzelter Fälle bekannt waren, war es verständlich, dass dieselben eine diagnostische Fehlerquelle in der erwähnten Richtung repräsentierten. Jetzt, da das Krankheitsbild durch eine Reihe Beschreibungen in allen seinen Phasen bekannt ist, ist die Möglichkeit es misszudeuten gering, und man wird immer darüber klar sein, dass Schlüsse aus dem Aussehen der Papille auf pathologische Zustände anderer Art bei dem Vorhandensein von Drusen nur mit grösster Vorsicht zu ziehen sind.

Die hier erwähnte Bedeutung, die den Drusen für die Diagnose von Sehnervenleiden zukommt, ist ja ganz negativer Art. Ob ausserdem einige positive Bedeutung in der klinischen Pathologie des Auges den Drusen zuzuschreiben sei, wird auf den Ergebnissen einer Untersuchung ihrer Ursachen und ihrer Wirkungen beruhen, hängt mit andern Worten davon $a b$, inwiefern man im stande ist, aus dem Vorhandensein ron Drusen in der Papille Schlüsse zu ziehen auf andere verursachende oder disponierende krankhafte Zustände im Auge oder im Organismus, und ferner, inwiefern Regeln anzugeben seien für den möglichen Einfluss der Drusen auf die Funktion des Auges.

Die histologischen Untersuchungen, die in einer Reihe von den veröffentlichten Fällen vorgenommen sind, haben keine sicheren Aufschlüsse über ihre Pathogenese gegeben. Wir. wissen, namentlich durch Cirinciones(22) und Sachsalbers (29) Untersuchungen, dass sie nicht von demselben Ursprung sind wie die Chorioidaldrusen, 
ferner dass sie aus einer Substanz gebildet sind, die mit Hyalin (v. Recklinghausen) identisch oder wenigstens sehr nahe verwandt ist, und endlich, dass sie den im Nervensystem - bisweilen im Nervus opticus und in der Retina - vorkommenden Corpora amylacea und hyaloidea in mehreren Richtungen, namentlich durch ihren geschichteten Bau, ähnlich sehen, ohne dass sie doch, namentlich wegen ihres stark lronglomerierten Auftretens, mit diesen Gebilden identifiziert werden können. Obwohl die Histologie also für das Verständnis der Natur der Drusen einige Anhaltspunkte gegeben hat, lassen sich doch hieraus gar keine Aufschlüsse über ihre Pathogenese gewinnen. Wie sie entstehen, ob das eigentliche Nervengewebe, das Stützgewebe oder die Gefässe das Material für ihren Aufbau liefern, darüber sind die Meinungen so divergierend wie nur möglich.

Von grösserem Wert für die Pathogenese sind die Aufklärungen, die sich aus den Krankengeschichten ausziehen lassen. Es zeigt sich nämlich, dass in einem sehr grossen Teil der beschriebenen Fälle die Drusen in Augen auftreten, in welchen ein anderer hrankhafter Prozess nachzuweisen und für das Auftreten der Drusen verantwortlich zu machen ist. Ich will hier eine kurze. Übersicht geben über die verschiedenen Leiden, die mit Drusenbildung im Sehnervenkopf kompliziert gefunden sind.

Retinitis pigmentosa wird gewöhnlich als das Leiden erwähnt, das relativ am häufigsten mit Drusen kompliziert wird; es kommt mir indessen etwas zweifelhaft vor, ob sie wirklich eine so dominierende Rolle in der Pathogenese spiele. Dass dies angenommen wurde, liegt darin, dass mehrere von den ersten darauf bezüglichen Mitteilungen Fälle solcher Art behandelten [H. Müller(1), A. Nieden (6), R. Ancke(16)]; in den zahlreichen später mitgeteilten Fällen ist aber Retinitis pigmentosa nur einmal notiert [Morton (35)]. Da die Fälle Anckes nur leichtere Grade von Drusenbildung umfassen, sind also Müllers, Niedens und Mortons Fälle die einzigen, in denen wohlentwickelte Drusenbildung beim betreffenden Leiden mit Sicherheit konstatiert wurde. Wenn Hirschberg $(14,22)$ das relativ häufige Vorkommen der Drusen als leuchtende Pünktchen im Umfange des Sehnerven bei Retinitis pigmentosa erwähnt, liegt wohl die Möglichkeit vor, dass solche leuchtende Pünktchen nicht mit Notwendigkeit als das Frühstadium wirklicher Drusenbildung anzunehmen seien.

Bei Chorio-retinitis pigmentosa atypica (nach Verletzung desselben oder des andern Auges im Kindesalter) wurden Drusen in 
der Papille von Oeller(8) und Remak(17) nachgewiesen; im Falle des letzteren scheint die Diagnose Drusen - wie früher erwähnt doch nicht ganz unzweifelhaft. Auch in Heyls (31) Fall bestand eine Chorio-retinitis mit Pigmentveränderungen im Augengrund.

Bei Neuritis optica und ihren Folgezuständen wurden die Drusen wiederholt gefunden, zuerst von Stood (9), später, von Wedl und Bock(18), Sachsalber(29) und Thomson(32). Wahrscheinlich dieselbe Ätiologie haben die Drusen in Schusters (41) Fall sowie in meinen Fällen I und IIT, vielleicht auch in Mendels (34) Fall. Schuster nimmt wohl an, dass die Drusen in seinem Falle ohne Entzündung entstanden seien, dies scheint mir aber widersprochen zu werden von den Einscheidungen der Gefässe, die eine nicht unbedeutende Strecke in der Retina zu verfolgen waren.

A trophia n. opt. (ohne sichere vorausgehende Neuritis) wird von Rath (20) und de Schweinitz (25) notiert. Auch in einem von Iwanoffs (2) Fällen, wo der geisteskranke Patient amaurotisch war, waren die Sehnerven zweifellos atrophisch.

Staungspapille als Ursache von Drusenbildung notiert Streiff (37) in zwei Fällen, während er in andern drei Fällen ohne sichere Anhaltspunkte dieselbe Ätiologie annimmt. In Hirschbergs und Birnbachers (15) Falle bestand ein beschränkter Prozess chronischer Stauung in den vorderen Abschnitten der Sehnerven.

Einseitige Drusenbildung nach einer vorausgehenden starken Läsion der betreffenden Schädelhälfte wurde in drei Fällen von Gessner(19) und Nieden(21) beobachtet. Da in zweien dieser Fälle Enophthalmus traumaticus bestand, irrt man sich wohl nicht in der Annahme, dass es sich hier um Orbitalfrakturen mit Läsion des N. opticus gehandelt hat.

Bei glaukomatöser Excavation wurden Drusen von Masselon(13), Terson(26) und Demaria (38) gefunden.

Läsion des Auges notiert Iwanoff(2) in drei Fällen, ohne die näheren Umstände zu erwähnen.

Ausserdem ist Drusenbildung in vereinzelten Fällen von Retinitis albuminurica, Gurwitsch (23), Iridochorioiditis supp., Schäfer(12), Chorio-retinitis syphilitica, Lawson(10) und Obliteratio art. centr. partialis, Story(11) wahrgenommen. Hirschberg (22) fand Drusen im Sehnervenkopfe bei Sarcoma chorioideae; da aber in diesem Falle im andern Auge, das sonst völlig normal ersohien, gleichfalls Drusen nachzuweisen waren, ist ihr Zusammentreffen mit Sarkom vermutlich als ganz zufällig aufzufassen. 
Es ist also eine ganz beträchtliche und recht bunte Reihe verschiedener Krankheiten, die mit Drusen kompliziert gefunden sind. Keine derselben scheint besonders stark vor den übrigen repräsentiert; Retinitis pigmentosa typica und atypica, Neuritis optica, Atrophia optica, Stauungspapille, Glaukom und Schädelläsionen kommen mit ungefähr gleicher Häufigkeit vor. Bei allen diesen Leiden ist es natürlich, die Drusen als das sekundäre Phänomen aufzufassen. Nur bei Glaukom ist eine andere Auffassung versucht, indem Masselon (13) die Vermutung aussprach, dass die Drusen durch Obstruktion der

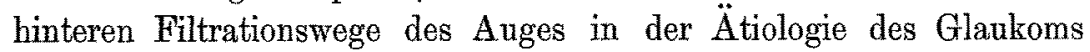
eine Rolle spielen könnten, ein Gedanke, der doch recht fernliegend scheint, namentlich wenn man berücksichtigt, dass niemals Zeichen von Drucksteigerung beim Vorkommen der Drusen gefunden sind, die Fälle ausgenommen, in welchen ausgesprochenes und weit vorgeschrittenes Glaukom vorhanden war.

Gemeinsam für alle die oben erwähnten Leiden ist offenbar, dass es sich stets um Zustände handelt, bei denen degenerative Prozesse im Nervengewebe des Sehnervenkopfes vorkommen oder sich voraussetzen lassen; in dieser Hinsicht einleuchtend scheinen mir namentlich die Fälle, in welchen die Drusen im Anschluss an Läsionen der gleichseitigen Schädelhälfte entstanden waren; hier könnten wohl als Ursache der Drusenbildung schwerlich andere krankhafte Prozesse in Betracht kommen, als gerade eine reine descendierende Degeneration des Nervus opticus infolge einer Läsion desselben. Entzündliche Vorgänge als notwendige Bedingung für Drusenbildung anzunehmen, so wie es Sachsalber (29) tut, scheint mir nicht zutreffend; in einem grossen Teil der Fälle kommen wohl Zeichen von Neuritis vor, es würde aber gekünstelt sein, neuritische Prozesse in allen Fällen anzunehmen, wogegen mehrere anatomische Befunde direkt sprechen. Die Drusen sind auch nicht während des Verlaufs einer Neuritis so frühzeitig gefunden, dass es nicht natürlich wäre, sie mit den der Neuritis folgenden degenerativen Zuständen in Verbindung zu setzen.

Während man also davon ausgehen kann, dass degenerative Vorgänge im Nervus opticus eine hervorragende Rolle in der Pathogenese der Drusen spielen, geht es doch gar nicht an zu schliessen, dass Degeneration des Nervengewebes eine notwendige Bedingung für ihre Entstehung sei.

Es ist eine recht grosse Reihe von Fällen mitgeteilt, in welchen die Drusen in Augen gefunden sind, die sonst keine deutlichen ob- 
jektiven oder funktionellen Zeichen von irgend welchem primären Leiden darboten. Dieses gilt von ungefähr $1 / 3$ von den mehr als 40 Fällen, die mir in genauen Beschreibungen bekannt wurden, und hierzu gehören mehrere von den genauest untersuchten Fällen und solche, die eine sehr bedeutende Entwicklung der Drusen aufwiesen. Wenn man die betreffenden Krankengeschichten mit Aufmerksamkeit durchgeht, bemerkt man wohl in einem Teil der Fälle Symptome, die deuten könnten auf die Möglichkeit von früher abgelaufenen krankhaften Prozessen im Auge oder von krankhaften Zuständen im Zentralnervensystem, die ja auf den Nervus opticus Einfluss gewinnen könnten. So wird Amblyopie seit frühester Erinnerung zweimal notiert [Nieden (21), Rabitsch (40)], anhaltende und starke Kopfschmerzen in zwei Fällen [Jany(7), mein Fall IV], Lähmung des Gesichtsnerven in einem Falle [Hirschberg(22)], und psychische Belastung mit hochgradigen neurasthenischen und asthenopischen Symptomen in einem Fall [Nieden(21)]. Indessen bleibt doch eine hinlänglich grosse Anzahl von Fällen, in denen nicht einmal solche Symptome nachzuweisen waren; als gute Beispiele hiervon lassen sich Niedens(21) und Laubers(44) Fälle und mein Fall II nennen. Es würde demnach nicht berechtigt sein, degenerative Vorgänge als notwendige Bedingung für Drusenbildung anzunehmen, selbst wenn man darüber klar ist, dass solche Vorgänge sich sehr wohl abspielen können, ohne die geringsten nachweisbaren Spuren in objektiver oder funktioneller Hinsicht zu hinterlassen.

Von den anatomisch untersuchten Fällen zeigten auch einer [Hirschberg und Cirincione(22)] nur so unbedeutende degenerative Veränderungen im Sehnerven, dass sie als Folgeerscheinung des Wachstums der Drusenmasse aufgefasst wurden; die übrigen zeigten ausser den Drusen auch andere krankhafte Zustände. Es ist also auch nicht mit Sicherheit bewiesen, dass die Drusen sich in einem völlig normalen Sehnerven entwickeln können.

Wie man sich auch zu dieser Frage stelle, steht es doch fest, dass die Drusen in ihrem Auftreten eine sehr grosse Selbständigkeit zeigen. Erstens ist es deutlich, dass der Entwicklungsgrad der Drusen in gar keinem Verhältnis steht zur Schwere des vorhandenen oder möglicherweise vorhandenen primären Leidens, da die ausgeprägtesten Formen sowohl mit als ohne gleichzeitige Zeichen von sonstigen Krankheiten

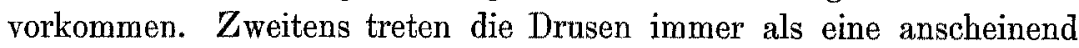
ganz zufällige und unberechenbare Komplikation auf, die im Verhältnis zur Häufigkeit der primären Leiden äusserst selten ist. 
Man wird deshalb zu der Annahme gezwungen, dass es andere Faktoren geben muss, die für das Zustandekommen der Drusen im Sehnervenkopf eine Rolle spielen, und es besteht demnach kein Hindernis anzunehmen, dass diese Faktoren auch in sonst normalen Papillen die Drusenbildung hervorrufen können, und dass also die erwähnten degenerativen Vorgänge nur disponierend oder veranlassend wirken.

Von welcher Natur diese pathogenetischen Faktoren seien, darüber geben die bisher beschriebenen Fälle nicht Gelegenheit, besondere Schlüsse zu ziehen. Dass die Disposition zu Drusen mitunter familiär und erblich sein kann, wird durch Anckes(16) und Laubers (44) Fälle bewiesen. Die Annahme Tersons (26), dass Altersveränderungen als Ursache anzunehmen wären, wenn nichts anderes für die Entstehung der Drusen verantwortlich gemacht werden könnte, ist leicht zu widerlegen, wenn man berücksichtigt, dass die Drusen grösstenteils bei jüngeren Individuen gefunden sind. In den Fällen, wo die Drusen in Augen ohne sonstige krankhafte Symptome nachgewiesen wurden, variiert das Alter von 15 bis 70 Jahren, mit dem Durchschnittsalter von ungefähr 38 Jahren; wenn man sich ausserdem erinnert, dass die Drusen sich sehr langsam entwickeln und sich nicht zurückbilden, ist der Gedanke, dass sie durchgehend im Anschluss an senile Veränderungen entstehen, ganz ausgeschlossen. In sämtlichen Fällen von Drusen des Sehnervenkopfes variiert das Alter von 11 bis 75 Jahren mit einem Durchschnittsalter von ungefähr 35 Jahren, also etwas geringer als in den unkomplizierten Fällen allein. Es geht indessen gar nicht an, in diesem Unterschied eine Stütze für Tersons Annahme zu suchen; denn erstens ist der Unterschied nicht grösser, als dass er auf Zufälligkeiten beruhen könnte; zweitens ist es ja wahrscheinlich, dass die Drusen beim Vorhandensein eines primären Leidens durchgehend auf einem früheren Stadium ihrer Entwicklung entdeckt werden, weil die Patienten wegen dieses Leidens unter Beobachtung sind zu der Zeit, in der die Drusen entstehen; beim Durchsehen der Fälle findet man in der Tat eine weit grössere Prozentzahl von vorgeschrittenen Fällen unter den anscheinend primären als unter den sekundären, die weit häufiger als jene in ihrem Anfangsstadium angetroffen sind; und drittens ist es ja möglich, dass die besprochenen primären Krankheiten die Entstehung der Drusen beschleunigen können bei Patienten, die mit Disposition zur Drusenbildung behaftet sind.

Eher als die Senilität scheint die Pubertät eine disponierende Bedeutung für die Entwicklung der Drusen haben zu können, indem 
sie nur in einem einzigen Falle [Gifford (30), ein 11jähriges Mädchen] vor dem 14. Lebensjahre nachgewiesen sind, während beginnende Drusenbildung sehr häufig - ja, am häufigsten - bei einem Lebensalter von ungefähr 20 Jahren angetroffen wurde. Indessen ist es nur in ganz seltenen Fällen möglich gewesen, den Zeitpunkt für den ersten Beginn der Drusenbildung festzustellen, und mit Rücksicht auf ihre sehr variable Wachstumsweise lassen sich gar nicht bestimmte Schlüsse daraus ziehen.

Betreffs des Geschlechts ergibt sich, dass in 30 Fällen die Patienten dem weiblichen angehören, in 23 Fällen dem männlichen. Dem Geschlecht ist also keine besondere Bedeutung in der Pathogenese zuzuschreiben.

Alkoholismus chronicus, vorausgehende Infektionskrankheiten und chronische Krankheiten verschiedener Art werden nur in ganz vereinzelten Fällen notiert und können also nicht als bedeutungsvolle ätiologische Faktoren angesehen werden.

Weitere Schlüsse über die Pathogenese der Drusen aus dem vorliegenden Material zu ziehen ist gewiss nicht möglich, und unsere Kenntnis von den Entstehungsursachen der Drusen ist also sehr mangelhaft. Will man sie in wenigen Worten ausdrücken, dann lässt sich wohl nur sagen, dass degenerative Prozesse im Seh. nervenkopfe in den meisten Fällen als mitwirkende Ursache nachzuweisen sind, dass eine familiäre Disposition in einigen Fällen vorkommt, und dass dem Pubertätsalter möglicherweise einige disponierende Bedeutung zuzu schreiben ist.

In praktischer Beziehung muss dies veranlassen, dass man den Fund von Drusen im Sehnervenkopfe als dafür sprechend, aber nicht beweisend für das Vorhandensein von degenerativen Zuständen in demselben ansieht; wenn es sich also um unsichere Diagnosen von krankhaften Prozessen im Nervus opticus oder im Zentralnervensystem. handelt, können die Drusen mitunter eine Stïtze für die Diagnose abgeben, ïbrigens ist aber ihr diagnostischer Wert offenbar gering.

Die andere Frage, die klinisches Interesse darbietet, ist die Frage über die etwaige Einwirkung der Drusen auf die Funktionen des Auges. Von vornherein sollte man ja glauben, dass so beträchtliche Veränderungen, wie die Drusen gewöhnlich darstellen, eine so massenhafte Anhäufung pathologischer Substanz in einem funktionell sehr wichtigen Gewebe zu sehr nachteiligen Folgen für das Sehvermögen führen müssten. 
Es scheint aber, als ob der Einfluss der Drusen auf das Funktionsvermögen des Auges sehr gering ist. In den Fällen, in denen Drusen als Komplikation bei andern Krankheiten auftreten, entspricht die Herabsetzung der Funktionen stets vollkommen dem Grade derselben; sichere Schlüsse lassen sich aber natürlich nicht ziehen aus den Verhältnissen in Augen, die auf andere Weise leidend sind.

Von grösserer Bedeutung für die Beurteilung sind die Fälle, in denen die Drusen sich als einziges krankhaftes Symptom zeigen, wenngleich auch hier grosse Vorsicht bei der Beurteilung zu verwenden ist, da vorausgehende Prozesse degenerativer Art ja niemals mit Sicherheit ausgeschlossen werden können.

In den 17 Fällen, die ich zu dieser Gruppe anführen kann, war die Sehschärfe in 12 Fällen ganz normal. In zwei Fällen [Jany (7), Morton(35)] war sie $6 / 6$ am einen, $6 / 9$ am andern Auge, die beide den gleichen Grad von Drusenentwicklung zeigten. In Niedens (21) erstem Falle war $S={ }_{i 5}$ an einem Auge mit excessiver Drusenbildung, während am andern Auge, das beginnende Drusenentwicklung zeigte, die Funktionen normal waren. In Niedens(21) zweitem Falle war Sehschärfe am einen Auge normal, während das andere Strab. diverg., Amblyopie seit der Kindheit und defektes Gesichtsfeld zeigte; die Drusen waren im gleichen Grade an beiden Augen entwickelt. In Purtschers (24) Falle bestand bei gleicher Drusenbildung an beiden Augen $S{ }^{6} \mid 9$ am einen Auge, während am andern (angeblich erst seit drei Wochen) $S$ auf Fingerzählen in 9,3 $\mathrm{m}$ reduziert war. Endlich war in Rabitschs (40) Falle bei starker doppelseitiger Drusenentwicklung $S=0,2$ am einen, 0,6 am andern Auge; die Amblyopie bestand angeblich seit frühester Jugend.

Das Gesichtsfeld, dessen Prüfung nur in wenigen Fällen ausdrücklich besprochen wird, zeigte gewöhnlich nichts abnormes ausser ganz geringen Defekten, wie leichte konzentrische Einengung der Grenzlinie für Blau in 2 von Niedens Fällen und ein geringer peripherer Defekt in meinem Fall II. Nur in den Fällen, wo die Sehschärfe sich als stärker herabgesetzt erwies, zeigte auch das Gesichtsfeld bedeutendere Defekte [Niedens(21) Fall 2, Rabitsch (40)].

In Niedens Fall 2 und in Rabitschs Falle ist die Amblyopie als angeboren oder jedenfalls sehr früh entstanden anzusehen, so wie auch die Verfasser es tun. In Purtschers Fall, wo die starke Amblyopie seit nur drei Wochen sich entwickelt haben sollte, lassen die Drusen sich nicht leicht für dieselbe verantwortlich machen, weil ihr 
immer sehr langsames Wachstum sich schwerlich mit einer so rapiden Schädigung des Nervengewebes in Übereinstimmung bringen lässt; von dieser Anschauung ist auch der Verfasser.

Es bleiben also nur einige ganz vereinzelte Fälle, in welchen die Drusen als Ursache einer ganz geringen Herabsetzung der Sehfunktionen angenommen werden können. Die besten Aufklärungen über die Verhältnisse gibt Niedens (21) Fall 1. Hier wurde das sehr bedeutende Wachstum der Drusenmasse mehrere Jahre hindurch genau beobachtet; doch trat keine bleibende Herabsetzung des Sehvermögens ein, indem dieses bei den ersten Untersuchungen zwischen $1 / 8$ und $2 / 3$ schwankte, während es schliesslich als $3 / 5$ notiert wurde. Dagegen wurden temporäre Herabsetzungen der Sehschärfe während der Exacerbationen im Wachstum der Drusen beobachtet; wenn aber ein Stillstand im Wachstum folgte, dann hob sich wieder die Sehschärfe.

Es scheint also, dass man als Regel festhalten kann, dass die Drusen nur selten und in geringem Grade das Funktionsvermögen des Auges beeinflussen; man muss annehmen, dass ihre Entwicklung so langsam und mit so geringer Energie vor sich geht, dass das Nervengewebe im stande ist, ihnen auszuweichen und sich den Verhältnissen zu accommodieren; hierauf deuten auch die mikroskopischen Befunde, die im ganzen Verschiebungen und nicht Zugrundegehen der benachbarten Nervenfibrillen gezeigt haben.

In ähnlicher Weise verhalten sich die Drusen den Gefässen gegenüber; diese werden von normalem Kaliber, aber oft verschoben und gebogen gesehen, und es muss also auch für sie angenommen werden, dass unter dem langsamen Wachstum der Drusen ihnen Zeit bleibt, sich den Verhältnissen zu adaptieren.

Es lässt sich also schliessen, dass die Prognose der Drusen im Sehnervenkopfe gut ist, und dass die Prognose der Leiden, die mit denselben sich komplizieren, durch diese Komplikation nicht verschlechtert wird. Wenn die Drusen in Verbindung mit einer stärkeren Verletzung der Funktionen oder mit den Zeichen von einer Cirkulationsanomalie auftreten, dann scheint es demnach auch berechtigt, solche Symptome als Zeichen aufzufassen, dass die Drusen sich im Anschlusse an ein anderes Sehnervenleiden entwickelt haben. 


\section{Literaturverzeichnis.}

(Die mit * bezeichneten Arbeiten waren mir nicht im Original zugänglich.)

1) Müller, H., Über Niveauveränderungen an der Eintrittsstelle des Sehnerven. A. v. Graef e's Arch. f. Ophth. Bd. IV, 2. S. 1. 1858.

2) Iw an off, Uber Neuritis optica. Sitzungsber. d. ophth. Gesellsch, im Jahre 1868. Klin. Monatsbl. f. Augenheilk. Bd. VI. S. 421.1868.

3) Liebreich. Ibid. S. 426. 1868.

4) v. Wecker, L. Graefe-Saemisch, Handb. d. ges. Augenheilk. Bd. IV. S. 641.1876 .

5) Leber, Th. Ibid. Bd. V. S. 910. 1877.

6) Nieden, A., Über Massententwicklung von Drusen der Lamina vitrea chorioideae nur im Umfange des intraokularen Sehnervenendes. Zentralbl. f. prakt. Augenheilk. Bd. II. S. 6. 1878.

7) Jany, Zur Kasuistik der Drusenbildung in der Lamina vitrea chorioideae an der Papilla nervi optici. Zentralbl. f. prakt. Augenheilk. Bd. III. S. 167. 1879 .

8) Deller, J. N., Beiträge zur Lehre von der Chorio-Retinitis pigmentosa. Arch. f. Augenheilk. Bd. VIIL. S. 435. 1879.

9) Sto od, W., Zwei Fälle über Drusenbildungen am intraokularen Sehnervenende. Klin. Monatsbl. f. Augenheilk. Bd. XXI. S. 506. 1883.

*10) Law son, G., Syphilitic choroido-retinitis with peculiar growths at the fundus. Transact. of the Ophth. Soc. of the Unit. Kingd. III. p. 117. 1883.

*11) Story, J. B., Anomalous distribution of retinal arteries. Transact. of the Ophth. Soc. of the Unit. Kingd. III. p. 102. 1883.

12) Schäfer, H., Anatomische Beschreibung eines Auges mit Irido-chorioiditis suppurativa. Zentralbl f. prakt. Augenheilk. Bd. VIII. S. 203. 1884.

*13) Masselon, Infiltration vitreuse de la rétine et de la papille. Paris 1884.

14) Hirschberg, Zentralbl. f. prakt. Augenheilk. Bd. VIII. S. 46. 1884.

15) Hirschberg u. Birnbacher, Beiträge zur Pathologie des Sehorgans VI. Zentralbl. f. prakt. Augenheilk. Bd. IX. S. 65. 1885.

16) Ancke, R., Beiträge zur Kenntnis von der Retinitis pigmentosa. Zentralbl. f. prakt. Augenheilk. Bd. IX. S. 167. 1885 .

17) Remak, Ein Fall von excessiver Drusenbildung in der Papille bei atypischer Retinitis pigmentosa. Zentralbl. f. prakt. Augenheilk. Bd. IX. S. 257.1885 .

*18) Wedl u. Bock, Pathologische Anatomie des Auges. S. 229. 1886.

19) Gessner, C., Enophthalmus traumaticus. Arch f. Augenheilk. Bd. XVIII. S. 297.1888.

20) Rath, W., Geschwülste der Hypophysis cerebri. A. v. Graefe's Arch. f. Ophth. Bd. XXXIV, 2. S. 81. 1888.

21) Nieden, A., Über Drusenbildung in und um den Optikus. Arch. f. Augenheilk. Bd. XX. S. 72. 1889.

22) Hirschberg, J., u. Cirincione, C., Über Drusen im Sehnervenkopf. Zentralbl. f. prakt. Augenheilk. Bd. XV. S. 166. 1891.

23) Gurwitseh, M., Über hyaline Bildungen im Sehnervenkopfe und in der Netzhaut bei Morbus Brightil. Zentralbi. f. pratt. Augenheilk. Bd. XV. S. 225.1891.

24) Purtscher, O., Drusenbildung im Sehnervenkopfe. Zentralbl. f. prakt. Augenheilk. Bd. XV. S. 292. 1891.

*25) de Schweinitz, G. E., Hyaline bodies in the nerve-head. Transact. of the Amerie. Ophth. Soc. VI. p. 349. 1892.

26) Terson, A., Les verrucosités hyalines de la portion papillaire du nerf optique. Arch. d'opht. XII. p. 367. 1892.

*27) Peters, Ein Fall von Drusenbildung am Sehnerven. Allgem. Zeitschr. f. Psych. Bd. L, 5. S. 1093.1894.

*28) de Schweinitz, G. E., Colloid disease in the macular region. Transact. of the Americ. Ophth. Soc. p. 212. 1894.

29) Sachsalber, A., Beitrag zur Drusenbildung im Sehnervenkopfe. Beiträge zur Augenheilk. Bd. XXI. S. 1. 1895. 
*30) Gifford, H., An unusual case of hyaline bodies in the optic nerve. Arch. of Ophth. XXIV. Nr. 3. 1895.

*31) Heyl, Albuminoid (?) deposit in the optic disc and retina. Transact. of the Amer. Ophth. Soc. p. 355. 1895.

*32) Thomson, E. S., Colloide Degeneration of the optic nerve. The PostGraduate. New York 1898.

33) Nieden, A., Drusenbildung des Optikus. III. Versamml. rhein.-westfäl. Augenärzte in Bochum am 3. Febr. 1900. Ref.: Zeitschr. f. Augenheilk. Bd. III. S. 361. 1900.

34) Mendel, F., Über Drusenbildung im Sehnervenkopfe. Berliner ophth. Gesellsch. 28. Juni 1900. Ref.: Zentralbl. f. prakt. Augenheilk. Bd. XXIV. S. 242.1900.

35) Morton, A. Stanford, and Parsons, J. Herbert, Hyaline bodies (Drusenbildungen) at the optic disc. Transact. of the Ophth. Soc. of the Unit. Kingd. XXIII. p. 135. 1903.

36) Hanke, Ein Fall von hyaliner Excrescenz der Chorioidea. Wien. ophth. Gesellsch. 9. Dez. 1903. Ref.: Zeitschr. f. Augenheilk. Bd. XI. S. 86. 1904.

37) Streiff, J. J., Über die Entstehung der Optikusdrusen. Klin. Monatsbl. f. Augenheilk. Bd. XLII, 1. S. 149. 1904.

38) Demaria, B., Zur Pathogenese der Amotio chorioideae nach Iridektomie bei Glaukom und über Corpora amylacea in der excavierten Papille. Klin. Monatsbl. f. Augenheilk. Bd. XIII, 1. 1904.

*39) Cirineione, Concrezioni nella testa del nervo ottico. La clinica oculist. 1904.

40) Rabitseh, F., Zur Kenntnis der Drusen im Sehnervenkopf. Klin. Monatsbl. f. Augenheilk. Bd. XLIII. S. 72. 1905.

41) Schuster, P., Zur Kasuistik krystallähnlicher Gebilde des Auges. II. Ein Fall von Drusenbildung im Sehnervenkopf. Arch. f. Augenheilk. Bd. LIV. S. 364. 1906.

*42) Harman, N. Bishop, Hyaline Degeneration of the Optic Disc. Transact. of the Ophth. Soc. of the Unit. Kingd. 14. Dez. 1905.

43) Bonh off, Bericht über die Wirksamkeit der Universitäts-Augenklinik zu Giessen. Inaug.-Diss. Giessen 1906.

44) Lauber, Ophth. Gesellsch. in Wien, 16. Jan. 1907. Ref.: Zentralbl. f. prakt. Augenheilk. Bd. XXXI. S. 144. 1907. 


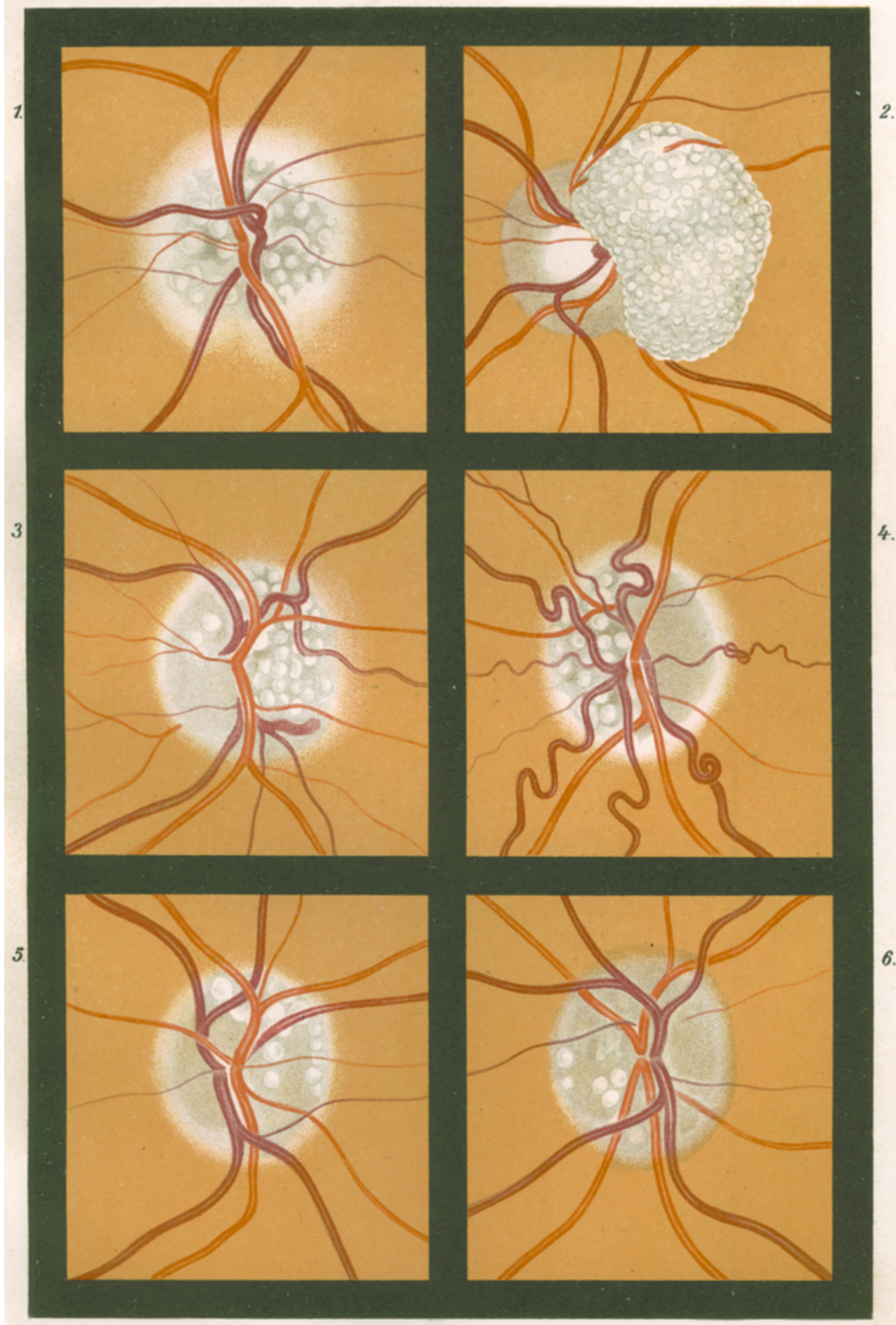




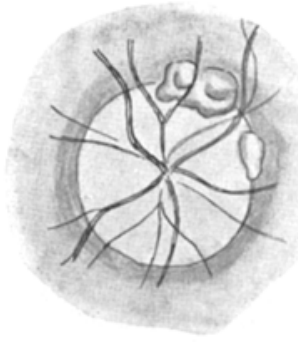

Fig: 7.

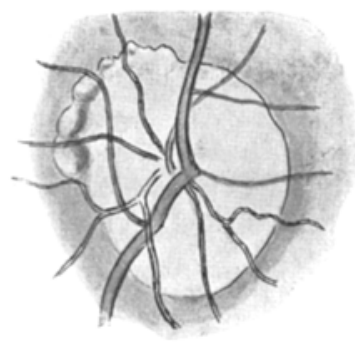

Fig. 8.

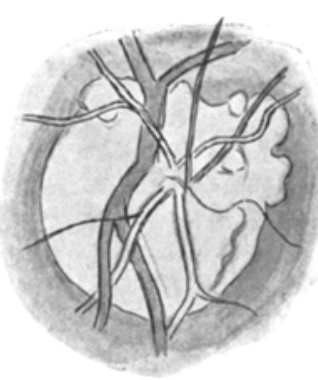

Fig. 9.

Hirschberg, Ctbl. f. pr. Au. 1891 . Sachsalber, Beitr. z. Au. 1895. Sachsalber, Beitr. z. Au. 1895.

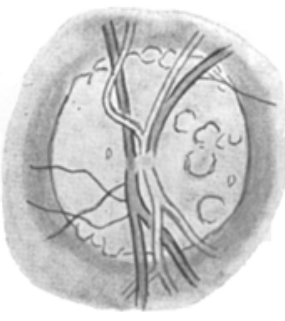

Fig. 10.

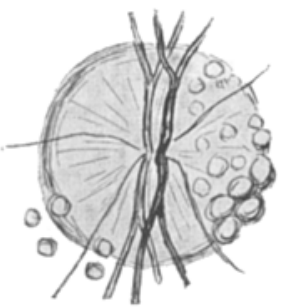

Fig. 11 .

Stood, KI. Mbl.f. Au. 1883.

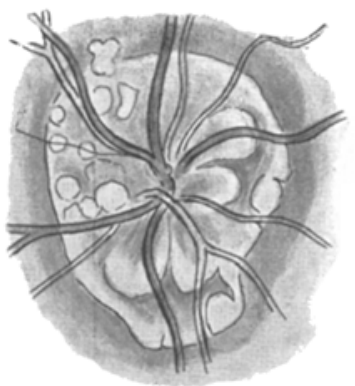

Fig. 14.

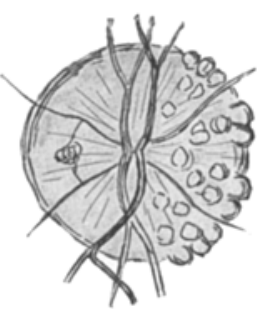

Fig. 12.

Stood, KI. Mbl. f. Au. 1883.

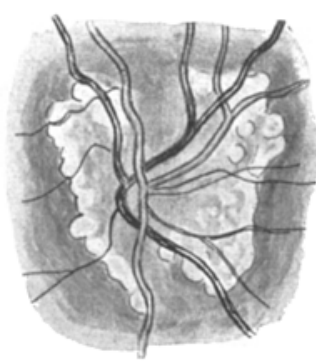

Fig. 15.

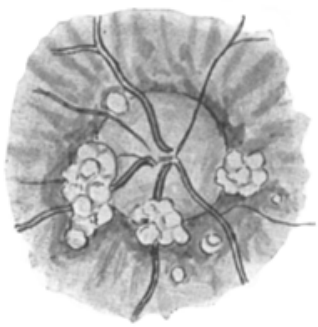

Fig. 16.

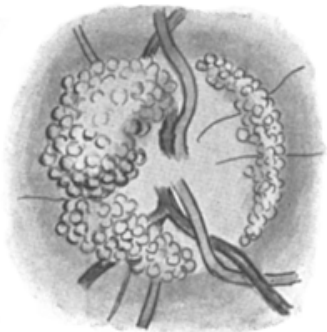

Fig. 17.

Morton, Oph. Soc. Un. Kgd. 1903. Nieden, Arch. f. Au. 1889.

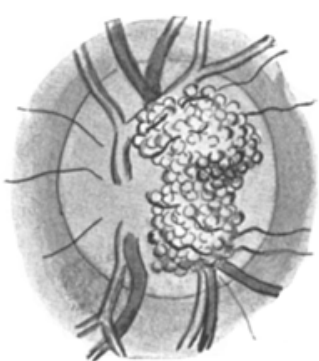

Fig. 18.

Nieden, Arch. f. Au. 1889. 